PREPARED FOR SUBMISSION TO JHEP

\title{
Quark and Gluon Jet Substructure
}

\author{
Jason Gallicchio, ${ }^{a}$ Matthew D. Schwartz ${ }^{b}$ \\ ${ }^{a}$ Department of Physics, University of California, Davis, CA 95616, USA \\ ${ }^{b}$ Jefferson Physical Laboratory, Harvard University, Cambridge, MA 02138, USA \\ E-mail: jason@frank.harvard.edu, schwartz@physics.harvard.edu
}

ABstract: Distinguishing quark-initiated jets from gluon-initiated jets has the potential to significantly improve the reach of many beyond-the-standard model searches at the Large Hadron Collider and to provide additional tests of QCD. To explore whether quark and gluon jets could possibly be distinguished on an event-by-event basis, we perform a comprehensive simulation-based study. We explore a variety of motivated and unmotivated variables with a semi-automated multivariate approach. General conclusions are that at $50 \%$ quark jet acceptance efficiency, around $80 \%-90 \%$ of gluon jets can be rejected. Some benefit is gained by combining variables. Different event generators are compared, as are the effects of using only charged tracks to avoid pileup. Additional information, including interactive distributions of most variables and their cut efficiencies, can be found at http://jets.physics.harvard.edu/qvg. 


\section{Contents}

1 Introduction $\quad 2$

2 History and Future of Quark/Gluon Measurements 4

$\begin{array}{lll}3 & \text { Theoretical Considerations } & 7\end{array}$

4 Event Generation $\quad 10$

5 Overview of Observables $\quad 11$

6 Evaluation of Discrimination Power $\quad 14$

7 Discrete (Particle/Track/Subjet) Variable Results $\quad 16$

8 Jet Shapes and Geometric Moments 19

8.1 Jet Mass 19

8.2 Traditional Jet Shape 19

8.3 Radial Geometric Moments 21

8.4 Linear Radial Geometric Moment: Girth, Width, or Jet Broadening 21

8.5 Jet Angularities $\quad 22$

8.6 Optimal Kernel for Radial Moment 23

8.7 N-subjettiness 24

8.8 Two-Point Moment 26

8.9 Two-Dimensional Geometric Moments 27

8.10 Pull 28

9 Combining Variables $\quad 29$

10 Comparing Variables $\quad 30$

11 Using Impure Samples to Verify Underlying Pure Distributions 35

12 Choosing the Operating Point for a Mixed Background 36

13 Comparing Herwig ++ to Pythia8 $\quad 39$

14 Conclusions $\quad 41$ 


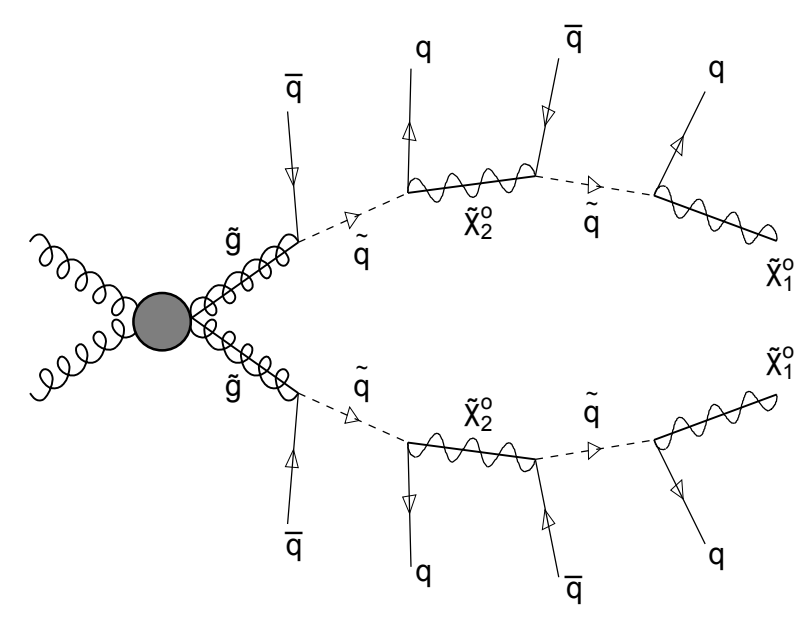

Figure 1. Gluino decay as an of of a quark-heavy signal, in this case with 8 quark jets and no gluon jets produced. Multi-jet events in standard model backgrounds are extremely unlikely to have so many quark jets.

\section{Introduction}

Being able to distinguish quark-initiated from gluon-initiated jets reliably at the LHC could be very useful, since signatures of beyond-the-standard-model physics are often quark heavy. For example, a typical gluino-pair production topology is pictured in Figure 1. Produced in pairs, each gluino's cascade decay can produce four quarks and missing transverse momentum due to the escape of the lightest supersymmetric partner. Backgrounds to this process have events with many jets produced from QCD. These jets are predominately gluonic. Additionally, many $R$-parity violating SUSY models produce quark jets without the missing transverse momentum. To constrain these models, being able to filter out background QCD events containing gluon jets would be helpful. Leptophobic $Z^{\prime}$ or $W^{\prime}$ particles provide other obvious examples where quark/gluon discrimination would be useful.

Gluon-heavy backgrounds are especially problematic for signals without leptons, gauge bosons, $B$-jets, tops, or missing energy. Quark/gluon tagging might be one of the few ways to improve these searches. Another application is to reduce combinatorial ambiguity within a single event. If jets in a given event could be identified as quark or gluon, their place in a proposed decay topology could be constrained, or they could be classified as initial-state radiation. Examining the quark/gluon tagging scores of jets produced by a new particle might be the only way to measure QCD quantum numbers directly. Alternatively, some signals consist of gluon jets, like coloron models [1] or buried-Higgs, where $h \rightarrow 2 a \rightarrow 4 g$ and $a$ is CP odd scalar [2]. The same observables and techniques apply to gluon tagging, though here we will treat the quark jets as the signal and the gluon jets as background for concreteness. 


\section{Chance EACH Jet is Quark}

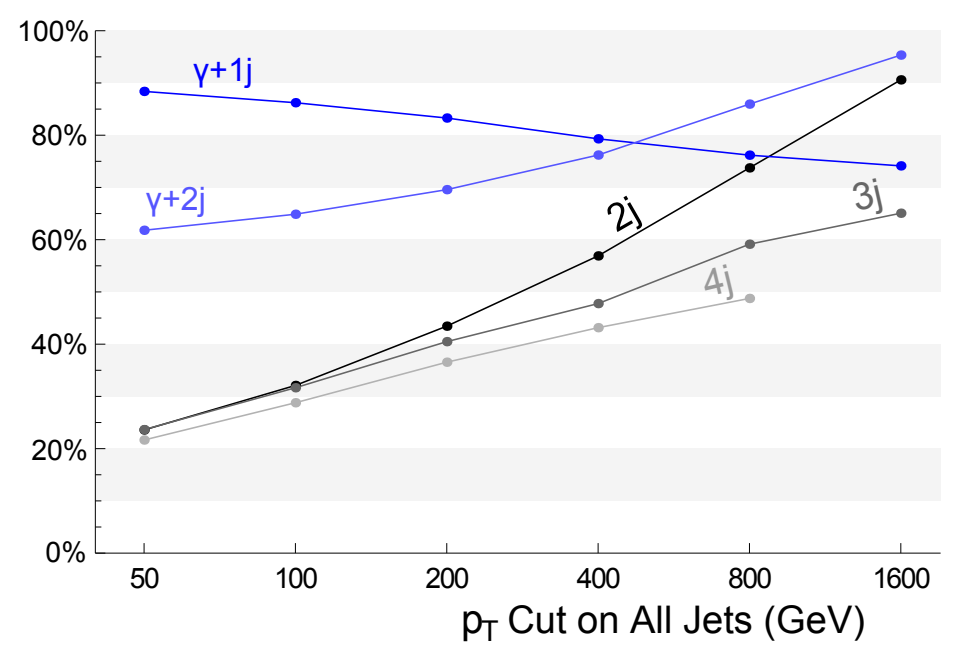

Figure 2. Fraction of jets which are a light quark jet (up, down or strange) rather than a gluon jet Here all jets have the minimum $p_{T}$ cut indicated, but photons have a minimum $p_{T}$ of only $20 \mathrm{GeV}$.

Practical quark/gluon discrimination would also be useful for some standard model studies. For example, in vector boson fusion (VBF), the forward jets are always quark jets whereas in non-electroweak backgrounds to $\mathrm{VBF}$, the jets near the beams are often gluonic. In the standard model, as the $p_{T}$ of jets increases, or if they are produced along with an electroweak boson, the fraction becomes more quark-heavy. This is shown in Figure 2. Thus, knowing the quark-to-gluon jet fraction of an event can help determine what are the underlying hard partons, with applications even in the standard model.

Differences between quark and gluon jets were measured in great detail in LEP 3-jet events, where the flavor could be known to high accuracy. Such measurements are described well by perturbative QCD calculations and leading-log parton showers combined with hadronization models. In the LHC era, we propose using this accumulated wisdom as a tool to find new physics. The small differences between generators do not invalidate the use of these tools to find observables that can distinguish between quark- and gluon-initiated jets on a jet-by-jet basis. Experimental effort can then be focused on the small set of the most powerful discriminators.

The goal of this hadron-level Monte Carlo study is to find properties of jets that best distinguish ones initiated by a quark from those initiated by a gluon. Charged particle count and jet mass are well known examples, but new observables like $p_{T}$-weighted moments as measured from the jet center and subjet properties provide additional handles. Each observable is examined for many jet $p_{T} \mathrm{~s}$, and the best set is combined into a quark/gluon tagger. Given a jet of a particular $p_{T}$, our tagger assigns a quark/gluon likelihood score. This can be cut on to purify the flavor content, combined with prior quark/gluon fraction into a 
true probability, or used in conjunction with $b$ and $\tau$ tagging scores to more fully classify the jet's flavor. We do not expect quark/gluon tagging to reach the same power as $B$-tagging or $\tau$-tagging, but our quark-efficiency vs gluon rejection curve can serve as a first approximation of what is achievable.

The main result of this paper is that a small set of 1-3 input observables capture nearly all of the quark/gluon differences. The most useful variables could become the focus of theoretical study, experimental measurement, and Monte Carlo validation. The multidimensional distributions of quark/gluon discriminating variables can be experimentally verified, for example, by looking at many samples with different known quark/gluon compositions, especially ones that are relatively pure [3].

Since jet properties depend strongly on $p_{T}$, We examine jets in narrow $p_{T}$ windows around six central values between $50 \mathrm{GeV}$ and $1.6 \mathrm{TeV}$, in powers of 2 . As a result of our examination of so many observables, we can make general statements about some $p_{T}$ trends.

For example, track counts are more useful at high $p_{T}$, whereas geometric moments (which measure the width/girth of jets) are more useful at low $p_{T}$. In addition, some observables are more powerful discriminants when the operating point of the tagger is chosen at high quark efficiency, and others are useful when a stronger cut is used to achieve high quark purity.

In the next section, we review past calculations and collider measurements at LEP and the Tevatron. After that, we define our observables, show hadron-level distributions, and quantify their performance. Finally we combine observables using boosted decision trees to form a multivariate discriminant. The final sections include comments on how one might use a quark/gluon tagger in situations where the signal or background contains both quark and gluon jets.

\section{History and Future of Quark/Gluon Measurements}

There are several differences between quarks and gluons that prove useful in motivating observables that can distinguish between the jets initiated by quarks as compared to gluons. Below is a list of properties and observables they motivate:

- Color Charge: $C_{F}$ vs $C_{A} \rightarrow$ jet mass, girth/width, track count

- Color Connections: 1 vs $2 \rightarrow$ eccentricity, planar flow, and pull

- Electrical Charge $\rightarrow$ charge-weighted track $p_{T}$

- Spin: $1 / 2$ vs $1 \rightarrow$ correlations in the location of subjets

An excellent review of theoretical and experimental results as of 2003 are presented in [4], some of which we now summarize. LEP studied the difference between quark and gluon jets by looking at 3 -jet events. These correspond to $e^{+} e^{-} \rightarrow q \bar{q} g$ at the parton level. At high center-of-mass energy, the two hardest jets are quark-initiated $99 \%$ of the time, thus one can use energy to select a pure sample. In another selection method, the highest energy jet is 
assumed to be a quark jet and one of the other jets is tagged for heavy flavor, which indicates the third should be gluonic. Alternatively, for three jets of similar energy, two $B$-tags gave a clean sample of $\sim 30 \mathrm{GeV}$ gluon jets.

LEP measured the ratio of the number of particles in gluon vs quark jets. The average multiplicity of any type of particle, along with its variance are given by the semi-classical approximation

$$
\frac{\left\langle N_{g}\right\rangle}{\left\langle N_{q}\right\rangle}=\frac{C_{A}}{C_{F}} \quad \frac{\sigma_{g}^{2}}{\sigma_{q}^{2}}=\frac{C_{A}}{C_{F}}
$$

where $C_{A} / C_{F}=9 / 4$. The angular width of the jet, using Sterman-Weinberg definition, is to leading order

$$
\delta_{g}=\delta_{q}^{C_{F} / C_{A}}
$$

An intuitive explanation for these results is that a quark jet is dominated by the first gluon emission, at which point it continues to shower like a gluon jet. Since gluon jets have more particles, for a given energy they will have correspondingly fewer hard particles.

In cases where QCD estimates do not agree with full simulation or with data, the reason is often attributed to energy conservation not being taken into account in each splitting. Since shower Monte Carlos enforce this energy conservation, they often have better agreement with data than the analytic estimates. Multiplicities have been calculated, including energymomentum conservation, at $\mathrm{N}^{3} \mathrm{LO}[5,6]$. At LEP I energies, the result was $\left\langle N_{g}\right\rangle /\left\langle N_{q}\right\rangle \approx$ 1.7. OPAL [7] studied the charged particle multiplicity in light quark jets of average energy $45.6 \mathrm{GeV}$ and gluon jets of $41.8 \mathrm{GeV}$. Agreement in the moments (mean, width, skewness, kurtosis) of the particle-count distributions was found to agree with the Monte Carlo event generators and with analytic predictions.

Subjet multiplicities were also examined at LEP for various subjet sizes [8, 9]. Extremely small subjets $\left(k_{T}=0.1 \mathrm{GeV}\right)$ approach the limit of particles, and therefore probed hadronization. But larger subjets $\left(k_{T}=5 \mathrm{GeV}\right)$ probed the better modeled, perturbative physics and gave the largest ratio between quark and gluon subjet multiplicities. For the first study cited, the average energy of the quark jets was $32 \mathrm{GeV}$, while that for gluon jets was $28 \mathrm{GeV}$. Later in this paper, we show that smaller subjets always improved quark/gluon discrimination at the LHC, down to the smallest subjets we probed with a resolution-limited size of $R_{\text {sub }} \approx 0.1$.

The particle types identified within jets also differ between quarks and gluons. For example, the numbers of $K^{0}, \Lambda, \pi^{ \pm}, K^{ \pm}, p, \eta, \eta^{\prime}$, and $\pi^{0}$ particles have been studied. LEP found an increase in baryons (protons and Lambdas) for gluon jets and an increase in kaons for quark jets, though $Z$ decays to $s \bar{s}$ or $c \bar{c}$ will have a higher strange-quark content than LHC's $u$ and $d$-dominated quark jets. Hadron identification within jets is reviewed in [4], where table 12.1 lists results of many LEP experiments. Some of the most relevant include DELPHI [10, 11], and OPAL [12, 13] measurements of identified particle ratios. We do not consider variables based on particle type, since their use depends strongly on how well these can be experimentally measured. 
Many of the LEP measurements were performed on the $Z$ pole, where the jets studied come from the decay of an on-shell particle (the $Z$ boson). These jets may not be typical of jets produced from QCD at hadron colliders. For example, the particle multiplicity can be computed in the $Z$ rest frame and is therefore independent of jet energy. In contrast, the particle multiplicity for a QCD jet at a hadron collider grows with the jet's energy.

LEP studies found $B$-jets to be more similar to gluon jets than to light quark jets $[14,15]$. The number of particles was higher in $B$-jets than in light quark jets, as was the angular spread. Both of these effects are due to the longer decay chain of $B$-hadrons, which overwhelms the effect of perturbative parton shower. The peculiarity of $B$-jets should be lessened in the LHC, which has higher $p_{T}$ jets and more boosted $B$-hadrons: for higher $p_{T}$, the QCD shower produces more particles, whereas the particle multiplicity is relatively fixed in the $B$-hadron decay. When the decay products of a $B$-hadron are specifically removed from consideration, the properties of $B$-jets will again look more quark-like.

Importantly, $B$-taggers rely largely on impact parameters or a secondary vertex, and so their efficiency should be largely uncorrelated with the observables we consider, which are constructed from the momenta of the particles. Thus, $B$-mistag rates are not significantly different for quarks as compared to gluons. One can imagine a 3-dimensional tagger based on the probability that a jet is either $b$, quark or gluon initiated. Since $B$-tagging is very dependent on experimental issues, we do not attempt to consider such a tagger here. We note that gluon splittings to heavy flavor, $g \rightarrow b \bar{b}$, are included in our simulation of gluon jets.

Compared to LEP studies using 25 to $45 \mathrm{GeV}$ jets, the LHC will typically have higher energy jets. Since high-energy jets are of particular interest to new physics searches, we consider jets with energies up to $1.6 \mathrm{TeV}$ in this study. At high energy, it is helpful to use longitudinally boost-invariant measures like transverse momentum and rapidity as opposed to energy and angle. Sometimes this motivates new variables appropriate to hadron colliders by replacing the LEP variable $E$ by $p_{T}$ and $\theta$ by $r=\sqrt{\Delta y^{2}+\Delta \phi^{2}}$.

Measures of the angular width of jets were used in DØ [16] and later CDF [17] to reject gluons and purify fully-hadronic top-quark samples. This may have been the first experimental application and proof that a separation exists in a complex hadronic collider environment. The CDF study showed that quark and gluon jets can be calibrated in a "naturally pure" quark sample (semileptonic tops without any explicit quark/gluon tagging).

Quark/gluon tagging should be even more useful at the LHC than it has been at LEP or the Tevatron. Compared to CDF and D $\varnothing$, ATLAS and CMS have better tracking and calorimeters, with spatial resolutions up to 10x as high. CMS's particle flow and ATLAS's individually calibrated TopoClusters give jet substructure techniques new power (especially if associated with the primary vertex and corrected for magnetic field bending). Also the LHC's proton-proton initial state, higher energy, and higher luminosity make gluon jets more common and more new physics signals are buried under multi-jet events. In addition, we find that higher $p_{T}$ jets of the LHC are more taggable than lower $p_{T}$ jets of previous colliders. For example, the charged track count becomes a better indicator of flavor as the jet $p_{T}$ increases. 


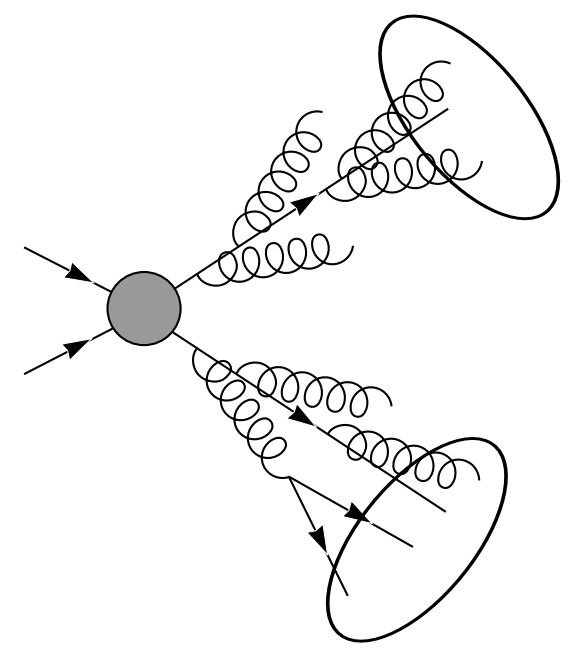

Figure 3. Jets are formed by grouping together collinear radiation.

\section{Theoretical Considerations}

Before cataloging and evaluating jet observables, it is worth commenting on the extent to which jet flavor is well-defined. We will argue that in the case of well-separated jets, appropriate for kinematic reconstruction, each jet can be assigned an unambiguous flavor. In other words, any situation which is problematic for quark/gluon tagging is also problematic for kinematic reconstruction. Thus, quark/gluon tagging is no more poorly defined than reconstructing a decay chain or other short-distance interpretation of an event with jets.

In the parton shower picture (which is in excellent agreement with data) a hard parton, well-separated from other hard partons in the event, undergoes showering that produces nearly collinear radiation. An example is illustrated in Figure 3. With reasonable assumptions about hadronization, any infrared and collinear-safe jet algorithm returns jets whose momenta correspond in some way to the initial hard partons. This parton/jet correspondence is implicitly assumed in all searches which use the resulting 4-vectors to reconstruct heavy objects like $W$ bosons, Higgs bosons, and tops. Violating any of these assumptions erodes the parton/jet correspondence.

For example, the shower products from two nearby hard partons could significantly overlap. Depending on the jet algorithm used, the jets might merge or have strange shapes. In such a case, the resulting jet momenta might not be useful for kinematic reconstruction and the jet properties (charged particle count or mass) might not be distributed in a way that corresponds to isolated quark or gluon jets.

One cause of unease is a sense that NLO quantum effects invalidate the semiclassical parton-shower picture. Much of the NLO corrections comes from real emission diagrams. At the quantum level, there is indeed interference between diagrams with the same final particle flavor and momenta. This is illustrated in Figure 4 where in one diagram a collinear 


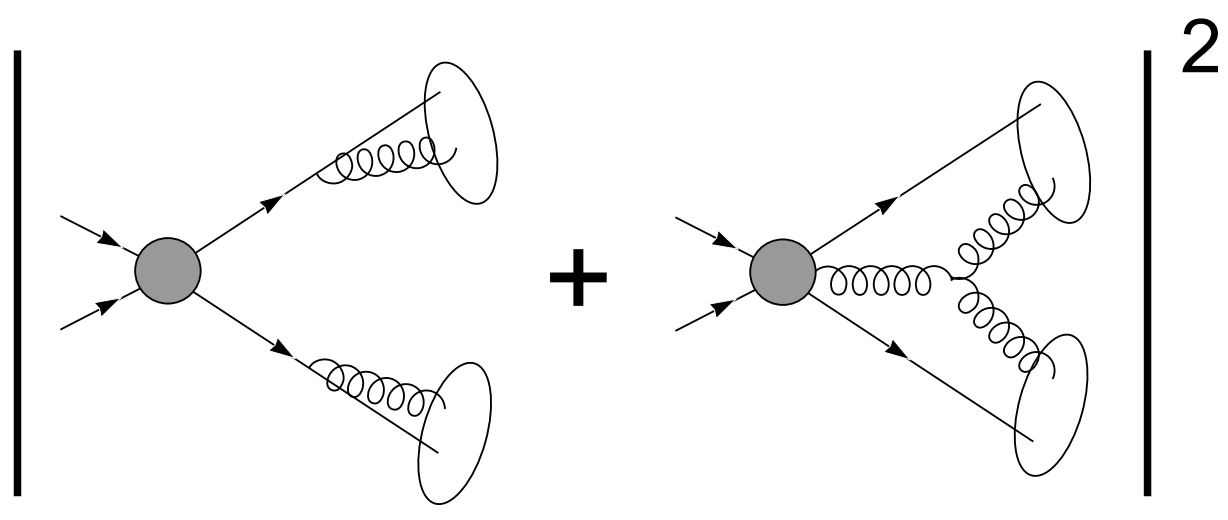

Figure 4. Parton showers produce quark jets whose properties are largely determined by the emitted gluons, as indicated in the left diagram. On the right, the same configuration is produced when a third hard parton, in this case a gluon, splits into two gluons with momenta equal to the showered gluons. Since the two amplitudes interfere, it might not make sense to describe this final state configuration as having two quark jets. In this case, however, the amplitude for the shower diagram is much larger than the hard-gluon-splitting diagram for the same final-state kinematics. In fact, as the gluons become more collinear with the quarks, the first amplitude is divergent.

gluon emission affects the properties of unambiguously quark-initiated jets, whereas the other diagram is a quantum mechanically indistinguishable correction where the gluons come from a completely unrelated additional hard parton. Looking only at the flavor and momenta of the final state, one might be uncomfortable claiming the configuration corresponds to two quark jets. However, the parton-shower-like, nearly collinear diagram has a much larger amplitude and therefore the uncertainty on labeling the configuration as having quark jets is small.

Up to an overall normalization, much of the NLO effects are reproduced by including matrix element corrections merged with a parton shower. In a fully-matched sample (using CKKW[18] or MLM[19] for example), each jet comes unambiguously from exactly one hard parton, and the flavor of this parton is known. The matching procedures have some merging scale, on which the final distributions depend only weakly. Thus one can make the same conclusion about matching for quark and gluon discrimination as for almost any other application (such as kinematic reconstruction): it gives unambiguous answers when the final state contains clearly separated jets. In ambiguous final states, which can be explicitly avoided, there is no well-defined underlying parton topology relevant for any analysis.

Ambiguities are always present in event-reconstruction from jets: fully hadronic $t \bar{t}$ decay doesn't always produce six clean, well-separated jets with unambiguous correspondence to $b$ and $W$ decay products. Thus, the problem is no worse for quark/gluon tagging than for top-reconstruction. Secondly, the mixing effect is numerically quite small. It is of course suppressed by a factor of $\alpha_{s}$. But also, hard splittings which change quarks to gluons or vice versa are power suppressed, for example by $m_{\text {jet }} / E_{\text {jet }}$. NLO ambiguities are important for 
measurements like the inclusive jet cross sections, but the bottom line is that NLO effects do not prevent a quark/gluon tagger from being a practical tool for many new physics searches.

For additional justification, we point out that $B$-tagging is at least as ambiguous as quark/gluon-tagging, and has been well-proven to be useful. For $B$-tagging, there are many simple, leading-order $B$-jet definitions. For example, $B$-jets can be defined as jets that contain a $B$-hadron among its decay products. This does not mean that a perfectly accurately $B$ tagged jet has a momentum that corresponds to the initiating $b$ quark. For example, an event with a $Z$ decaying to $b$ and $\bar{b}$ quarks doesn't necessarily produce a pair of $B$-tagged jets that have an invariant mass that corresponds to the $Z$. Away from the mass peak, either the wrong jets contained the $B$-hadron or there is simply no single jet that corresponds to each $b$ quark. At the Monte Carlo level, the hard $b$-quarks won't correspond to $B$-tagged jets any better than a $W$ boson's direct quark decay products will correspond to two jets that should obviously be labeled the quark jets.

An alternative to using the event record in a Monte-Carlo to extract truth information about the hard parton initiating a jet would be to cluster the partons in the jet such that flavor information is retained. An algorithm for doing so was proposed by Banfi, Salam, and Zanderighi in [20]. Their idea was to count the number of quarks minus anti-quarks in a jet. By itself, this would not be a good infrared and collinear-safe definition. But they modified the $k_{T}$ jet-clustering algorithm to combine only partons that preserve flavor in an infrared and collinear-safe way. Gluons can be combined with $u$-quarks to make a a $u$-jet, $u$ and $\bar{u}$ can be combined into a flavorless gluon-jet, but $u$ and $d$-quarks cannot be combined. The focus of [20] was on precision calculations in perturbative QCD with a small number of partons involved. The applications of quark/gluon tagging at hadron colliders are somewhat different. Since the observables at colliders are tracks and calorimeter deposits from color-neutral hadrons, a parton-level jet-flavor algorithm like the one in [20] is not directly applicable. The exact quark minus anti-quark count is not reliably observable, nor does it directly capture the useful but vague notion that a particular jet 'was initiated by' a particular quark or gluon. The algorithm in [20] could be used on the pre-hadronization partons in a Monte Carlo event record to assign a truth-flavor in a non-matched sample. But if the relevant hard partons are available in the event record, one might as well use them for the truth information, since this corresponds exactly to what one is trying to extract from the event.

To verify the distributions of the variables discussed below, samples of known flavorcomposition can be used. For example, in a $\gamma j j$ event when the softer jet is near enough to the photon, it is over $98 \%$ likely to be a quark jet. (This can be understood from the simple observation that quarks radiate photons but gluons do not.) A catalog of high cross-section processes and kinematic cuts which can be used to purify samples was given in [3]. The fraction of a cross section consisting of quark or gluon jets is in fact well-defined beyond the leading order in perturbation theory as long as the jets are hard and well-separated. This follows essentially because helicity is conserved in the collinear limit, as discussed in detail in [3]. 
Jets from the pure samples discussed above might not be representative of jets in a signal or background of interest. To predict the jet properties of a new signal, simulations must be employed at some level, and there are problems assigning truth-level flavor to these jets. One popular method is to 'match' the jets to the hard process using their $\Delta R$. While this is common, it doesn't take into account how well the energies match. It is also not guaranteed, for example, that the 4-momenta of hard partons from MadGraph are preserved when PYTHIA adds initial state radiation and has to rebalance the event. This procedure can only be trusted in a matched sample, where the hardest jets have explicit matrix-level counterparts. Another method is to examine the shower history, which can be used to assign a jet a truth tag if a large enough fraction of its energy is 'descended' from a single hard parton. Changing the definition of 'large enough' might alter the distribution of the jet properties of interest. But a larger problem is that soft radiation is really a dipole effect, sourced by two hard partons, whereas PYTHIA randomly assigns this soft radiation to either one or the other parent.

\section{Event Generation}

Most of the results in this paper pertain to generated with MADGRAPH V4.4.26 [21] and showered through PyTHIA V8.140 [22] with most recent default tune. We also compare to the same events showered with HERWIG ++2.5 .2 [23]. Jets are reconstructed using FASTJET V2.4.2 [24]. The multivariate analysis is done using the TMVA V4.0.4 package [25] that comes with ROOT v5.27.02 [26].

No detector simulation was done. Instead, we discard charged particles with momenta less than $500 \mathrm{MeV}$. These particles are not allowed to contribute to either the construction of jets or the observables involving charged tracks. This $500 \mathrm{MeV}$ cut is identical to early ATLAS studies [27] (later studies have raised the cutoff to $1 \mathrm{GeV}[28]$ ). With data and better tunes, a full detector simulation (not publicly available) will become necessary to validate the various variables.

Since experiments also compare to Monte Carlo truth-hadrons, our study provides a useful rendezvous point. The goal of this paper is to point out potentially interesting observables, some new, which might either be used right away or studied in greater detail. To that end, we have made an effort to find observables that depend more on the perturbative parton shower than on hadronization. No effort has been made to explicitly consider multiple interactions, though they are included in the underlying event model. Pileup, however, is not explicitly included since removing it is best studied with a full detector simulation.

We start from a dijet sample $p p \rightarrow j j$ with the jets in each sample having their $p_{T}$ in windows centered around values spaced by factors of 2 in GeV: $(50,100,200,400,800,1600)$. We also considered a back-to-back $\gamma+$ jet sample for the same jet $p_{T} \mathrm{~s}$, and the results were nearly identical.

The shape of the quickly falling $p_{T}$ distribution within each window affects the efficiencies of various variables. For example, the $p_{T}$ of a jet depends on the jet algorithm and jet size; this dependence is precisely one of the variables studied here that usefully distinguishes quarks 
from gluons. That the cross sections fall sharply with $p_{T}$ makes the initial sample selections quite delicate. Ideally we would simulate a 'natural' dijet $p_{T}$ distribution and select jets only within an infinitesimal window around each $p_{T}$. With a narrow enough window, the falling distribution can have a negligible effect. But the shower, hadronization, and jet algorithms must all be run before this determination can be made, so an extremely narrow window is computationally inefficient.

To deal with the rapidly falling distributions, we chose parton-level MadGraph cuts to reproduce samples with 'natural' anti- $k_{T} R=0.5$ jet $p_{T}$ distributions within a $\pm 10 \%$ window, starting with the most narrow parton $p_{T}$ window that was possible. Even when quark and gluon partons start with an identical initial $p_{T}$, after showering, quark jets had to higher average $p_{T}$ than gluon jets. This was compensated by shifting and widening the initial parton-level $p_{T}$ windows to efficiently generate a representative distribution of jet $p_{T} \mathrm{~s}$ within the narrower jet window. The gluon and quark parton $p_{T}$ windows were chosen to have a $\pm 20 \%$ width and were shifted relative to each other to align the center of their anti- $k_{T}$ $R=0.5$ jet $p_{T}$ distributions on the nominal values. The resulting $p_{T}$ distributions still aren't exactly identical: the gluon has more of a lower tail, and the quark distribution has an uppertail. So only jets within $\pm 10 \%$ of the nominal $p_{T}$ were kept. Additionally, for dijets, the $p_{T}$ distribution of the gluons falls faster than that for quarks (as can be inferred from the changing fractions in Figure 2), but for our narrow final jet $p_{T}$ window, this slope difference is negligible. Our shifting and spreading successfully decoupled jet $p_{T}$ from the jet properties while maintaining somewhat efficient event generation. This prevents our tagger from picking up on the difference in $p_{T}$ distribution of the input samples rather than the jet properties.

This pre-shift and post-window procedure above slightly biases the sample for a finite width. Any 'real' set of jets at a particular $p_{T}$ will include some whose underlying parton was much softer, and others where it was much harder. A 'natural' $p_{T}$ distribution, especially for a QCD background, is exponentially falling, which means that jets at a particular $p_{T}$ will more likely come from softer partons that showered-up rather than harder partons that showereddown. With these caveats, the best advice is to take our scores as a rough guide, focus on ones that don't change drastically with jet $p_{T}$, and train any multivariate discriminant either bin-by-bin in $p_{T}$, or on the actual underlying $p_{T}$ distribution of your signal and background samples. To construct a general gluon tagger, the experiments will need a canonical jet definition so they can train it on a set quark and gluon jets with identical $p_{T}$ distributions with respect to that jet definition. Some anti- $k_{T} \mathrm{R}=0.5 p_{T}$ distributions within our windows are shown in Figure 5.

\section{Overview of Observables}

For the purposes of quark/gluon tagging, a jet can be thought of a set of particles, tracks, or calorimeter deposits. Each constituent has a 4-momentum and possibly a charge or particle ID, though this is difficult to determine. Given this huge set of constituent data, the goal is to estimate the likelihood that the jet was initiated from a quark rather than a gluon. 

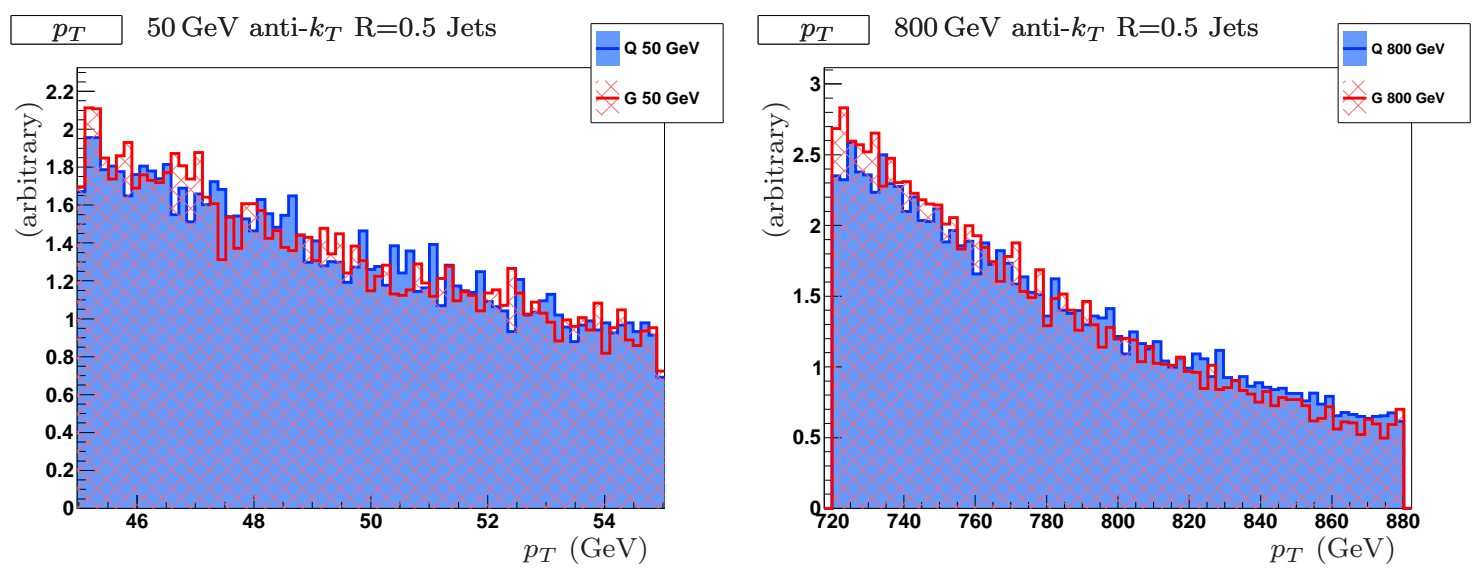

Figure 5. The $p_{T}$ distributions for two quark and gluon jet samples from PyтнIA 8 with arbitrary normalization. Our samples at each $p_{T}$ were chosen such that anti- $k_{T} \mathrm{R}=0.5$ jets had $p_{T}$ values within $10 \%$ of the nominal value. For all QCD jets, this distribution is falling, but within our window, the $p_{T}$ itself cannot be used distinguish quark from gluon jets. On the left is the $50 \mathrm{GeV}$ sample, and on the right is the $800 \mathrm{GeV}$ sample.

If a jet is made of 100 constituents, each with a massless 4-momentum, the problem is 300-dimensional. Ideally we'd have a fully differential cross section: a 300-dimensional probability density for quark jets, and one for gluon jets. This would take into account important correlations when reducing the list of particles to a one dimensional quark/gluon likelihood, but of course this is completely unrealistic. Familiar multivariate classifiers like Neural Networks or Boosted Decision Trees are designed to estimate this likelihood if properly trained. Unfortunately, they aren't designed to deal with a long, variable-length list of inputs. In other words, we can't just give them the 4-momenta for each particle in the jet and hope for the best. The challenge is to find simple observables that allow us to get as close to this ideal likelihood as possible. Since the particles are not independent, a good observable must extract the most important correlations.

As in jet algorithms, we'd like to do this in a way that is infrared and collinear-safe. This means that a jet's score shouldn't change if one particle in the jet is replaced by two where either (1) both are traveling in the same direction as the original or (2) one has very soft momentum. Raw particle count is not infrared safe, nor are things that depend directly on particle count like average particle $p_{T}$. Charged particle count with a minimum $p_{T}$ is safer with respect to soft emission and also safer with respect to collinear emissions, since these must conserve charge.

Infrared and collinear safety is usually framed as a strict yes/no requirement in the limit of exactly collinear splitting or zero momentum soft emission. In reality, by the time individual tracks or calorimeter deposits are observed, they would never be exactly collinear nor infinitely soft. Thus it is more meaningful to envision spectrum of safety. For example, while all of the popular iterative jet algorithms are infrared safe, counting the number of small 
anti- $k_{T}$ subjets of size $R=0.1$ is less safe than counting larger $R=0.3$ subjets. Unfortunately, the smaller subjets turn out to be more useful, and the charged particles even more so.

Thus we consider two main types of observables: ones that try to distinguish individual particles, tracks, or subjets, and ones that treat the energy or $p_{T}$ within the jet as a function of $(\delta y, \delta \phi)$ away from the jet axis.

The first category includes things like count, average $p_{T}$, and spread (standard deviation) in $p_{T}$ for these discrete objects. Subjets can be obtained by explicit $k_{T}$ declustering into $N$ jets, or by running a jet algorithm again with a much smaller $R$. These have been studied and confirmed extensively at LEP, and provide better discrimination at high quark efficiency and high $p_{T}$, but can be more difficult to measure at hadron collides in crowded jets. Here CMS particle flow or Atlas TopoClusters can extract the most information out of each jet. The identities of particles were not explored by us, but as mentioned above, LEP found an increase in baryons (protons and Lambdas) for gluon jets and an increase in kaons for quark jets.

The discrete-category observables that we evaluated are listed below. In later sections, the useful ones are described in more detail, distributions are shown, and gluon rejection scores are compared for different parameters (like jet size).

Distinguishable Objects (particles/tracks/subjets) (Section 7):

- Particle/track/subjet multiplicity, with different subjet algorithms and sizes $R_{\text {sub }}$

- $\left\langle p_{T}\right\rangle$ : Average $p_{T}$ of particles/tracks/subjets within the jet

- Higher statistical moments like $\left\langle p_{T}^{2}\right\rangle$ and $\sigma_{p_{T}}$

- Average distance from jet axis $\langle r\rangle$ and higher moments like $\left\langle r^{2}\right\rangle$

- $\left\langle k_{T}\right\rangle$ : Average $k_{T}$, the momenta transverse to the jet axis.

- The $p_{T}$ fraction or $\Delta R$ of subjets, explicitly $k_{T}$ declustered into $N$ jets

- Subjet count above a particular $p_{T}$ or a fraction of jet $p_{T}$

- Subjet splitting scale

- Masses of subjets

- Charge-weighted $p_{T}$ sum of tracks

The second, more continuous, category includes jet mass, jet broadening, and the family of radial moments. These tend to be better for lower $p_{T}$ jets and better at achieving highpurity at the cost of low quark-efficiency. Some observables we evaluated are listed below with more detail left to later sections.

Continuous Shapes (Section 8): 
- Jet Mass and $m / p_{T}$ ratio (Section 8.1)

- Jet Shapes: integrated and differential, to some distance from the jet axis (Section 8.2)

- Radial moments (Section 8.3)

- "Girth" of each jet (Section 8.4)

- Jet Broadening (Section 8.4)

- Jet Angularities (Section 8.5)

- Optimal Radial Moment (Section 8.6)

- N-Subjettiness (Section 8.7)

- Two-Point Moment (Section 8.8)

- Higher geometric moments like eccentricity or planar flow (Section 8.9)

- Pull as a measure of color-connections (Section 8.10)

When we describe these variables in more detail, it will be useful to have a way to score or rank them. In the next section, we describe our scoring methods.

\section{Evaluation of Discrimination Power}

In this section we describe ways of quantifying our jet observables' quark/gluon tagging power. We will then use gluon rejection at a fixed quark acceptance to rank our variables to find the most powerful discriminants. We will also look at how the discrimination power depends on things like jet $p_{T}$ or jet size.

One method of evaluating an observable's discriminating power is the separation [25]

$$
\left\langle S^{2}\right\rangle=\frac{1}{2} \int \frac{\left[\hat{p}_{S}(x)-\hat{p}_{B}(x)\right]^{2}}{\hat{p}_{S}(x)+\hat{p}_{B}(x)} d x
$$

where $\hat{p}_{S}(x)$ and $\hat{p}_{B}(x)$ are the signal and background probability density functions (PDFs) of some observable $x$. Identical distributions give zero separation, while ones with no overlap give a separation of one. This definition is invariant under any one-to-one change of variables. This invariance this fixes the exponent on the PDFs used in the numerator and denominator. This measure does not directly tell us how the observable will perform as a tagger, so we do not use it.

Other measures of discrimination power involve the so-called ROC curve ${ }^{1}$ shown in Figure 6 . The ROC curve is constructed by sliding a cut across the variable and plotting the

\footnotetext{
${ }^{1}$ The name comes from radar. It stands for Receiver Operating Characteristic.
} 

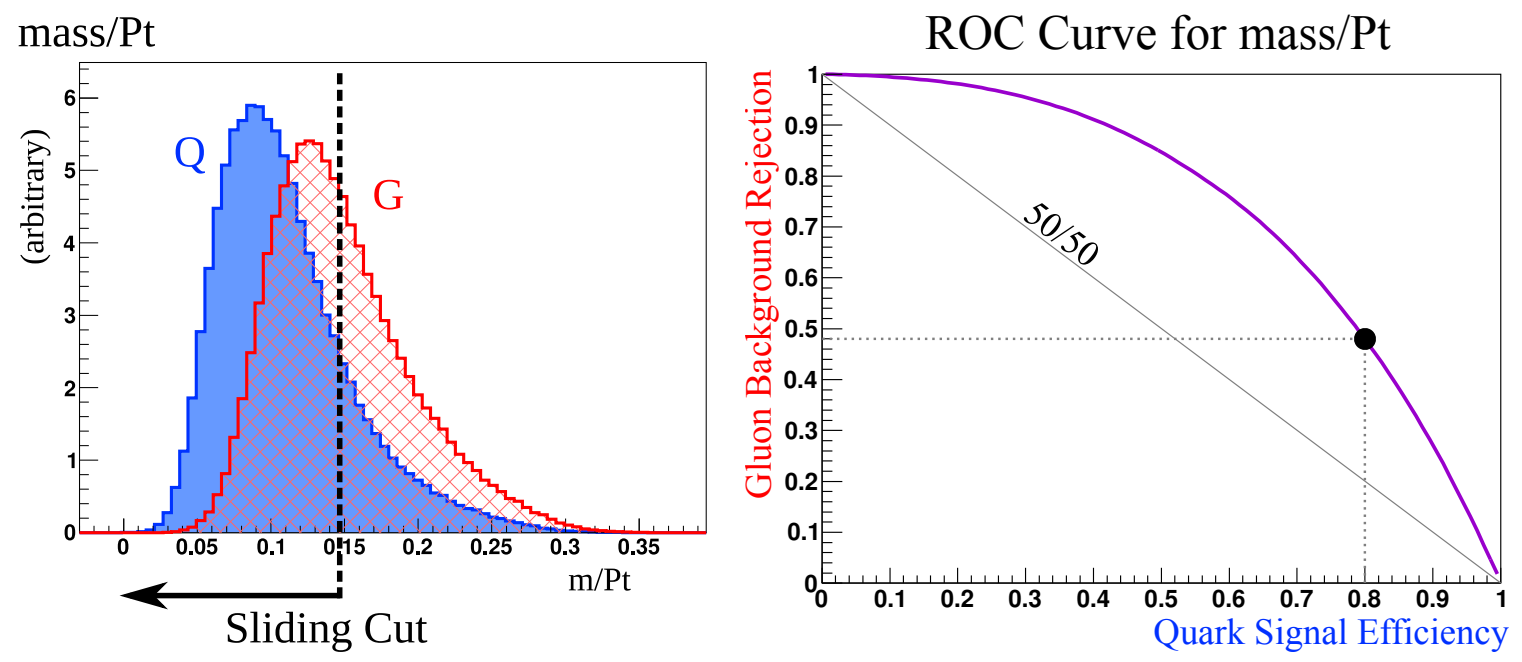

Figure 6. On the left is the distribution of PyтнIA 8 particle jet mass divided by $p_{T}$ for quark and gluon jets of $p_{T} \approx 200 \mathrm{GeV}$. In all plots that follow, quarks are always solid blue and gluons hashed red, and the distributions are arbitrarily normalized to equal area. Every cut leads to a particular efficiency for keeping quarks and a (hopefully lower) efficiency for keeping gluons. One minus this gluon efficiency is the background rejection. The curve formed by all possible cuts is called the ROC curve, and is shown on the right. The particular point shown corresponds to keeping $80 \%$ of the quark signal while rejecting $50 \%$ of the gluon background.

gluon 'background' rejection against the quark 'signal' acceptance. If the distributions completely overlapped, the result would be the diagonal line. Height above the diagonal represents discrimination power. One way to quantify this power is to choose a reference signal efficiency ( $80 \%$ is shown) and measure background rejection there. Variables can be ranked by rejection power at this chosen signal efficiency.

Variables with more complicated distributions might require a two-sided cut with the signal either inside or outside the cut. For two-sided cuts there is no unique background rejection for a given signal efficiency, so the best value is used. Even more complicated distributions, including multivariate ones, require more general boundaries (a contour in 2D, a surface in 3D, etc.) Transforming the (possibly multidimensional) quark and gluon observable distributions into a single likelihood $q /(q+g)$ distribution always allows for a single-sided cut on this likelihood. If the multidimensional distributions were known a priori, a sliding cut on this likelihood would form the best possible ROC curve. Any one-to-one map of this likelihood like $\log (q / g)$ can also be cut on to produce an identical ROC curve. A good multivariate discriminator (i.e. neural net or boosted decision tree) estimates one such one-to-one map given limited training data.

Sometimes ROC curves for different variables cross. We will find, for example, that at high signal efficiency (a loose cut), counting the charged tracks is the best observable, while for low signal efficiency (a tight cut), observables like jet broadening are best. In these 

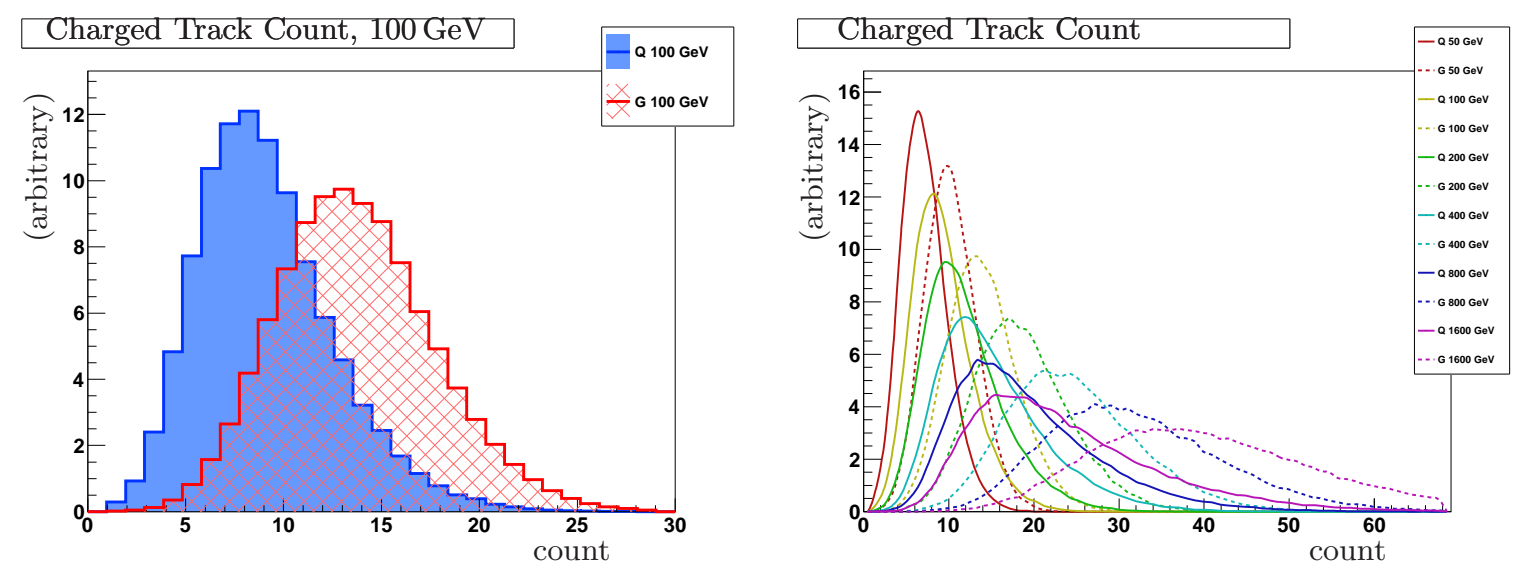

Figure 7. Charged track count for Pyтнін 8 anti- $k_{T} \mathrm{R}=0.5$ jets, normalized to equal area. Left: $100 \mathrm{GeV}$ jets with quark jets in solid blue and gluon jets in hashed red. Right: All $p_{T}$ samples where solid is quark and dotted is gluon. For a variable that is less sensitive to the jet $p_{T}$, the count could be divided by the log of the jet $p_{T}$.

situations, there is no unique way to rank the relative discrimination power since different variables are superior for different signal efficiencies. The area under the ROC curve, which is equivalent to averaging the rejection power over all signal efficiencies, provides another measure of discrimination power without having to pick a reference signal efficiency. Ranking the variables by area turns out to be very similar to ranking them by their background rejection at around 50\% signal efficiency, where the distance to the diagonal is greatest.

Any measure of the discrimination power of a variable is going to depend on the $p_{T}$ of the jet, along with other parameters like the jet algorithm or jet size. It will also depend on the source (PythiA, HeRwig, data), although robust and well simulated variables should minimize this dependence. For these reasons, we'll do our best to display and summarize our findings, but the final recommendation will always be subject to validation on real data.

\section{Discrete (Particle/Track/Subjet) Variable Results}

For discrete variables our results can be summarized simply as "smaller is better". Counting all particles gives the best discrimination power, although since neutral particles often cannot be distinguished, especially in a high-pileup environment, using all particles may not be practical. If all particles are not available, the next best option is counting charged tracks. We define charged tracks to mean all charged particles in the jet above $0.5 \mathrm{GeV}$. Distributions of charged track count is shown in Figure 7, and the gluon rejection for all $p_{T}$ samples, jet sizes, and three different quark efficiencies are shown in Figure 8.

After charged particle counts, the smallest subjets do best. Distributions for anti- $k_{T}$ $\mathrm{R}=0.1$ subjets are shown in Figure 9 . The rejection power of subjets of many types and 
Charged Track Count

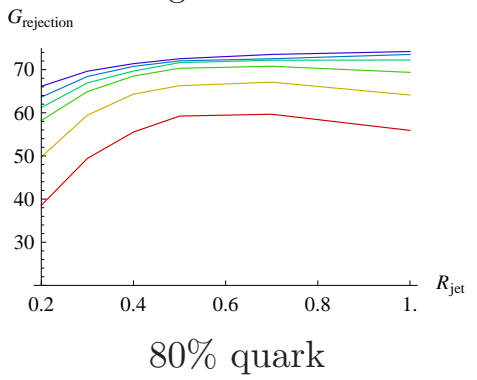

Charged Track Count

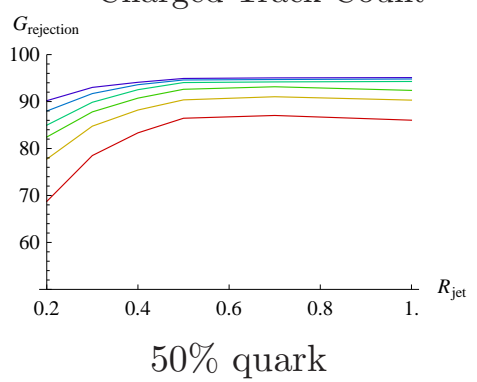

Charged Track Count

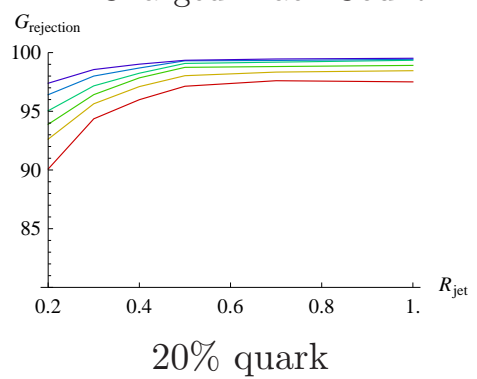

Figure 8. For a single variable, in this case PyтнIA8 charged track count, each panel is the gluon rejection for a different quark fraction. A mild $80 \%$ cut is shown on the left and the harshest $20 \%$ cut is on the right. Each plot shows the gluon rejection percentage (vertical axis) as a function of jet size (horizontal axis). The different lines in each plot correspond to different jet $p_{T} \mathrm{~s}$, with the red (bottom) being $50 \mathrm{GeV}$ and going by factors of two until the purple (top) at $1600 \mathrm{GeV}$. The vertical scale is different for each plot, but higher rejection is always better. Jet sizes between $R=0.5$ and $R=0.7$ achieve the best rejection. Similar to all count-type variables, higher $p_{T}$ jets can achieve better gluon rejection because the shower has more 'time' to establish the different particle counts.
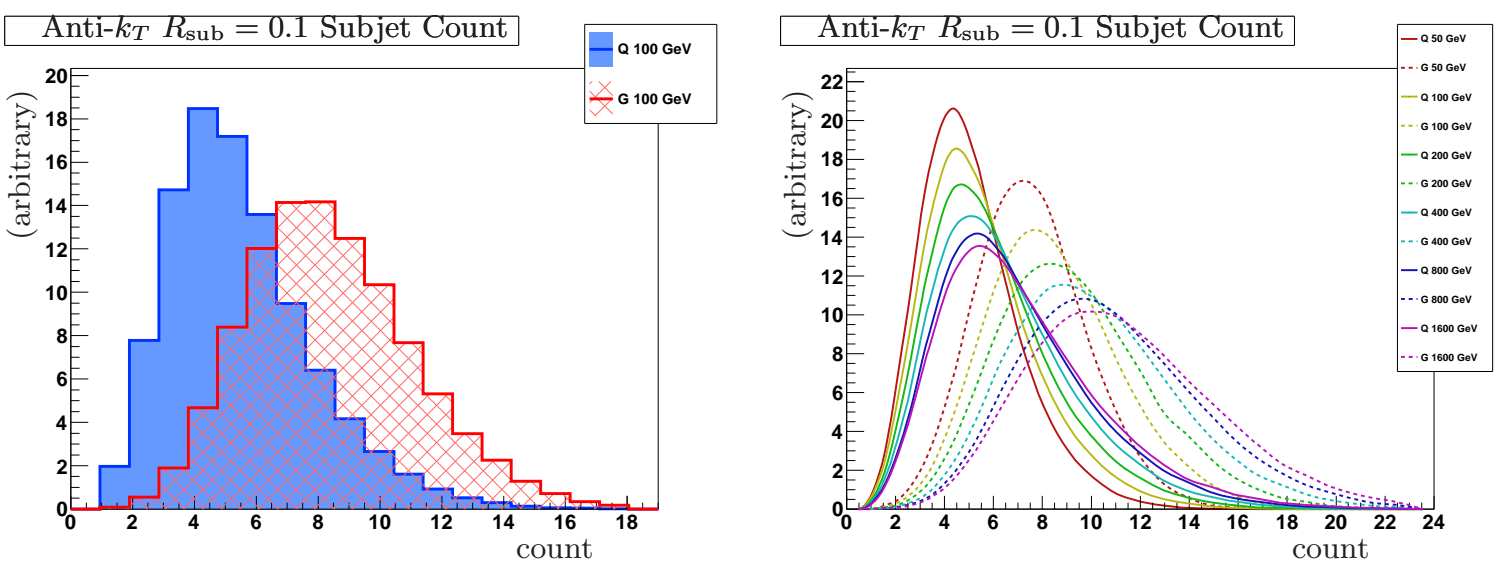

Figure 9. Subjet count for Pythia 8 anti- $k_{T} \mathrm{R}=0.5$ particle jets and subjets using anti- $k_{T} \mathrm{R}=0.1$, normalized to equal area. Left: $100 \mathrm{GeV}$ jets. Right: All $p_{T} \mathrm{~s}$.

sizes is shown in Figure 10. The smallest have $R_{\mathrm{sub}}=0.1$, the approximate resolution of distinguishable TopoClusters.

Finding the average particle/track/subjet $p_{T}$ and normalizing to the jet $p_{T}$ gives no more information than the count. Finally, the standard deviation of subjet $p_{T} \mathrm{~s}$ (also normalized by the jet $p_{T}$ ) is useful, but not more than the counts, and also not as useful when combined with other observables. It is not shown here. 


\section{Subjet Count}

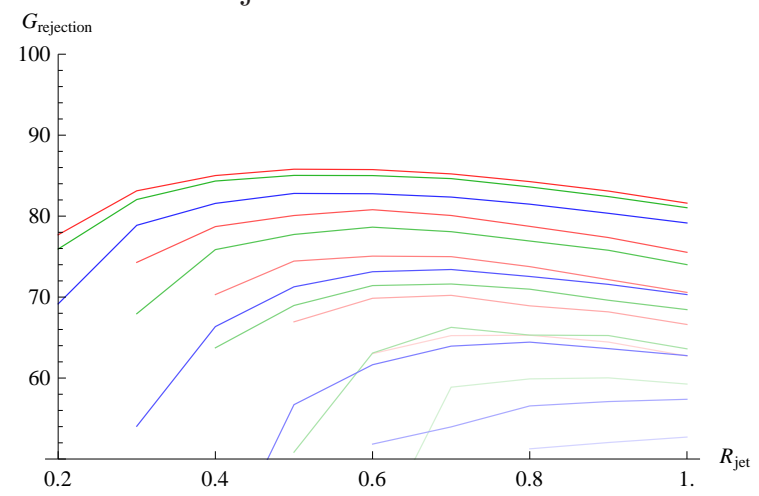

Subjet $p_{T}$ Standard Deviation

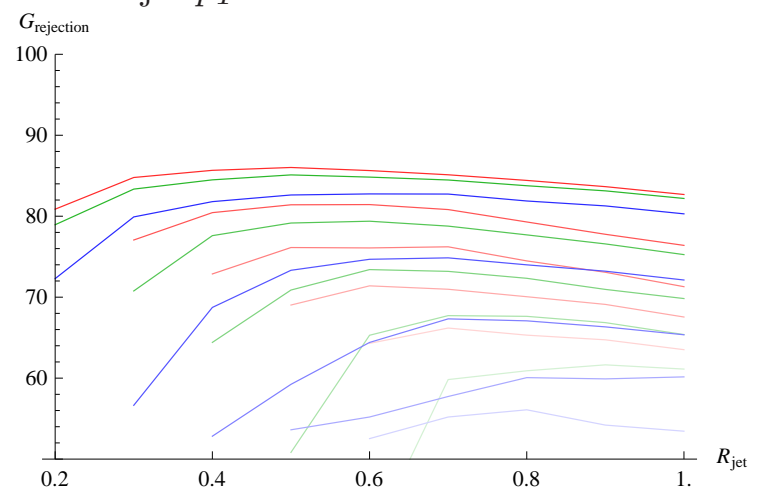

1st Subjet's $p_{T}$ Fraction

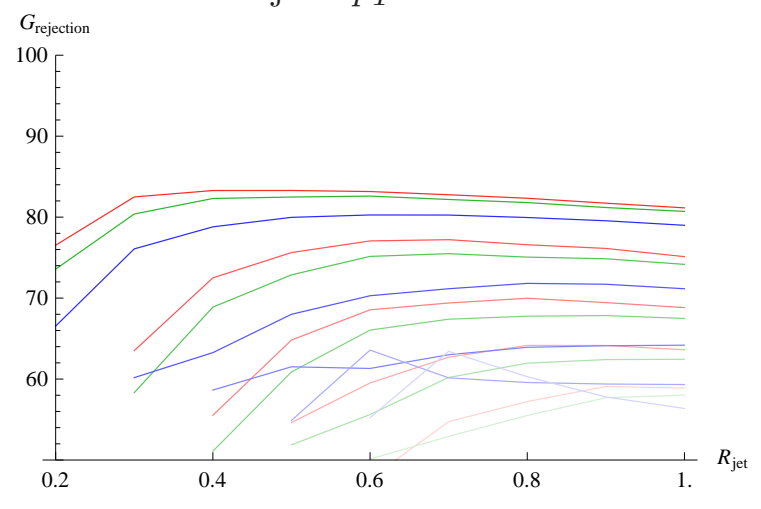

2nd Subjet's $p_{T}$ Fraction

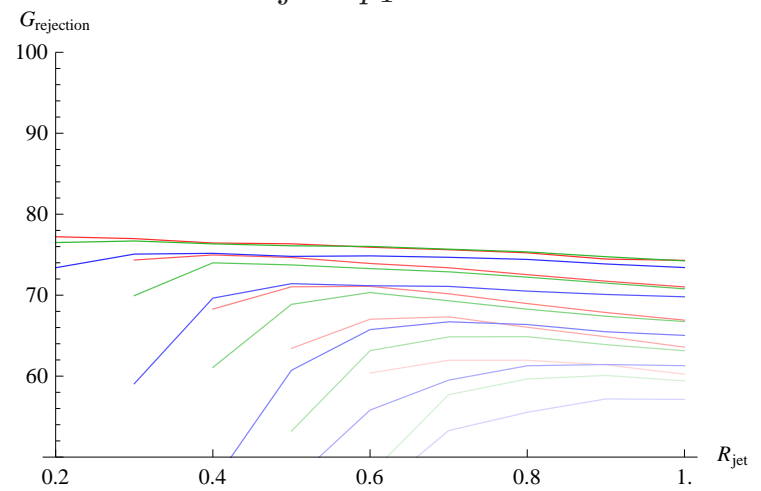

Figure 10. For subjets, smaller is better. Gluon rejection at 50\% quark acceptance is plotted as a function of initial jet size $R_{\text {jet }}$. These scores are averaged over all jet $p_{T}$ bins from $50 \mathrm{GeV}$ to $1600 \mathrm{GeV}$ for Pythia8 particle jets. The color corresponds to the subjet algorithm, with anti- $k_{T}$ in red being slightly better than $\mathrm{CA}$ in green, which is slightly better than $k_{T}$ in blue. As for subjet size, the darkest color corresponds to the smallest and best subjet size of $R_{\mathrm{sub}}=0.1$. Lightest is the largest and worst subjet size of $R_{\text {sub }}=R_{\text {jet }}$. These trends hold even for subjet variables not plotted. 


\section{Jet Shapes and Geometric Moments}

Unlike counting tracks or taking $p_{T}$ of the hardest small subjet, the continuous category requires more detailed definitions. We therefore provide explanations along with the results in this section. We first discuss the simplest jet shape, jet mass. We next discuss what is traditionally called the jet shape, including its integrated and differential versions. We then describe some useful variables like angularity and girth which are basically radial moments of jet shape. Next we consider more complicated observables like N-subjettiness and the moments of a two-point function. Finally we describe $2 \mathrm{D}$ moments in the $(\eta, \phi)$ plane like planar flow and pull.

\subsection{Jet Mass}

A jet's 4-vector is obtained by adding up the 4-vectors of all of the jet constituents. As long as the constituents are not collinear, the resulting jet 4 -vector will be massive. This jet mass measures how spread out the constituents of the jet are. Distributions of jet mass normalized to jet $p_{T}$ for different samples, along with their gluon rejection power is shown in Figure 11. There is already data [29] and theoretical calculations [30, 31] of jet mass at the LHC.

\subsection{Traditional Jet Shape}

The Jet Shape is an example of an IRC safe observable that is commonly used in jet-property studies. Each jet has its own integrated jet shape $\Psi(r)$, which measures the fraction of the jet's total $p_{T}$ that falls within $r$ of the jet axis. This is illustrated in Figure 12 and defined
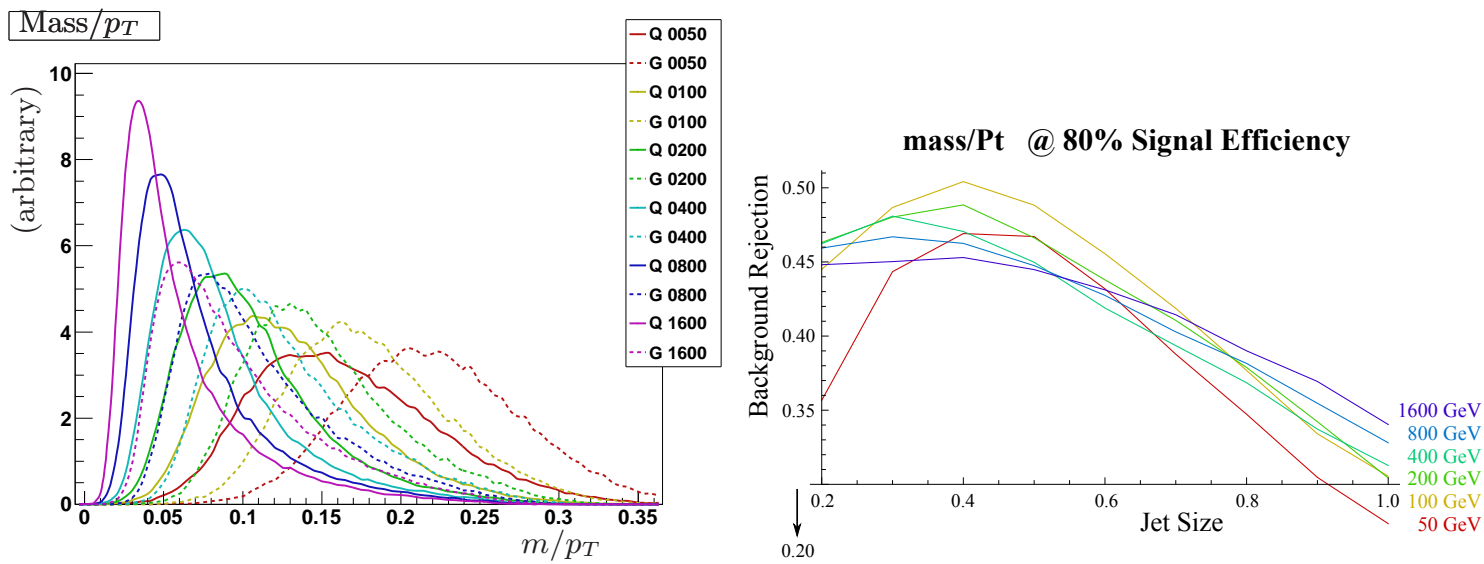

Figure 11. Jet mass over jet $p_{T}$ for Pythia 8 anti- $k_{T}$ particle jets. Left: Different jet $p_{T} \mathrm{~s}$ for $\mathrm{R}=0.5$ jets, normalized to equal area. Right: Gluon rejection scores as a function of jet size for different $p_{T}$ samples. Red is $50 \mathrm{GeV}$, yellow is $100 \mathrm{GeV}$, and so on, doubling through the spectrum until purple at $1600 \mathrm{GeV}$. The best gluon rejection occurs between $\mathrm{R}=0.3$ and $\mathrm{R}=0.5$. 

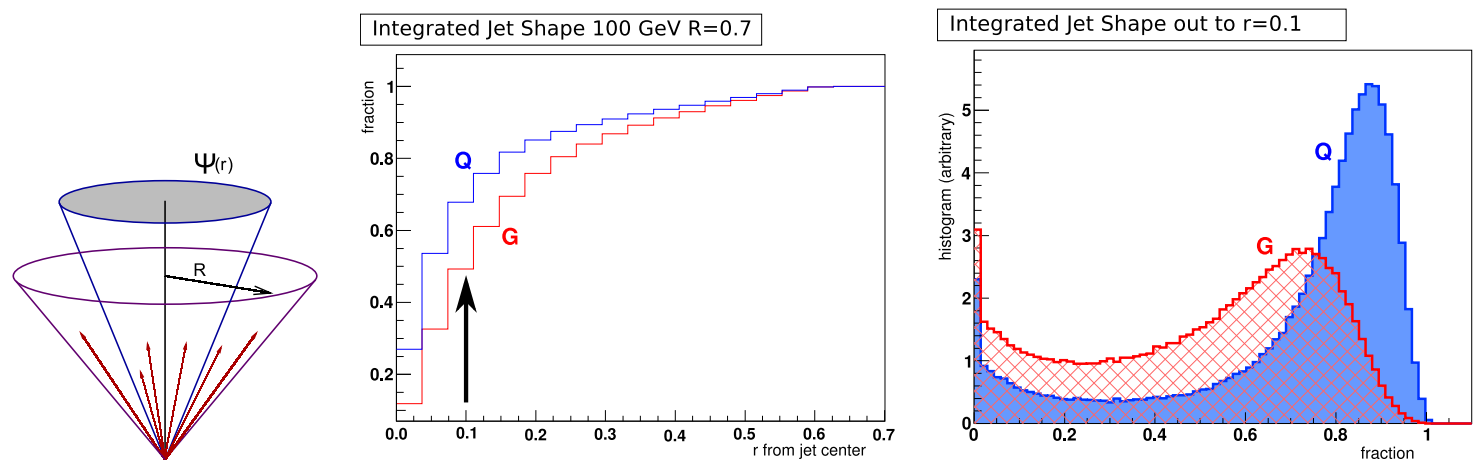

Figure 12. The Integrated Jet Shape $\Psi(r)$ is the fraction of the $p_{T}$ of a jet of cone size $R$ falling within a smaller cone of size $r$, as illustrated in the far left panel. $\Psi\left(r=R_{\text {jet }}\right)=1$ by definition. In the center is a plot of the integrated jet shape averaged over all observed jets of a particular type (here our Pyтнia8 quark and gluon dijet sample). On the right the distribution of $r=0.1$ jet shapes is shown. The mean of these distributions gives $\Psi(0.1)$ for quarks and gluons. The distribution is clearly not a simple Gaussian centered around the average value, indicating that much information is discarded in considering only the integrated jet shape. The rise at low $r$ is due to jets where the parton underwent a semi-hard splitting leading to little $p_{T}$ deposited along the jet axis.

more precisely as

$$
\text { Integrated Jet Shape: } \Psi(r)=\int_{0}^{r} \frac{p_{T}\left(r^{\prime}\right)}{p_{T}^{j e t}} d r^{\prime}
$$

An important distinction must be made between this definition, which is different function for each jet, and what is commonly plotted as 'jet shape,' which is averaged over all jets seen by a detector (with some cuts.) In Figure 12, this averaged integrated jet shape is the left plot, whereas the distribution of integrated jet shapes out to a single radius of $r=0.1$ is the right plot. The distribution is clearly not a Gaussian centered around the average value. Given $\Psi(0.1)$ for a particular jet that you want to classify, it's more useful to know the full distribution for quarks and gluons than just the two average values. Historic measurements and calculations are for the average rather than the full distribution. The same is true for jet masses: often average masses are calculated and measured for different $p_{T} \mathrm{~s}$ rather than mass distributions.

Measurements at CDF agreed well [32] with Pythia Tune A and Herwig out to $p_{T}^{\text {jet }}=$ $380 \mathrm{GeV}$. At higher $p_{T}$, shapes got narrower, which is consistent with the mix of quark and gluon jets evolving from $27 \%$ quark at $50 \mathrm{GeV}$ to $80 \%$ quark at $350 \mathrm{GeV}$. Early ATLAS data also agrees moderately well [27] with simulations. When used event-by-event, often a particular annulus was chosen to be integrated over, for example $0.2<r<0.7$ in the CMS jet shape briefs [33] and [34]. At the Tevatron, CDF chose $0.3<r<0.7$ [32]. This particular choices were not optimized for distinguishing quarks from gluons. 


\subsection{Radial Geometric Moments}

We refer to any geometric moment that is linear in $p_{T}$ and independent of angle around the jet axis as a radial moment. Linearity in $p_{T}$ is required for IR/collinear safety. Specifically, the $p_{T}$ in each radial bin is weighted by a kernel $f(r)$ and summed up to form the moment $M_{f}$ :

$$
\text { Radial moment using kernel } f(r) \quad M_{f}=\sum_{i \in \text { jet }} \frac{p_{T}^{i}}{p_{T}^{j e t}} f\left(r_{i}\right)
$$

Distances $r$ of each particle or cell from the jet center are calculated on the (rapidity,phi) cylinder. The jet center is taken as the $(y, \phi)$ of the jet's 4 -vector, but the $p_{T}$-weighted centroid is almost identical. It is important to use rapidity rather than pseudorapidity for the jet location because the jet is massive. A radial moment sums a function of these distances, weighted by $p_{T}$, then normalized to the total $p_{T}$ of the jet. Energies and angles, rather than $p_{T} \mathrm{~s}$ and $r$ 's give similar results, but are less appropriate to hadron colliders.

The integrated jet shape $\Psi(0.1)$ corresponds to the moment where $f(r)$ is 1 out to $r=0.1$ and 0 beyond. The differential jet shape $\psi(0.3)$ corresponds to a kernel that is 1 in a small window around $r=0.3$. One series of kernels are powers of $r: r, r^{2}, r^{3}, \cdots$. These most closely correspond to the traditional geometric notion of 'moments.' Radial moments like these are interesting because it may be possible to calculate them accurately in QCD, see for example [35].

An orthonormal set of kernel functions fully characterizes the radial distribution of $p_{T}$ for a single jet, but even knowing the 1D distributions for an infinite set of orthogonal functions would not give complete information about the underlying high-dimensional distribution with all correlations preserved. In other words, knowing this series for a particular jet would allow a full reconstruction of where the $p_{T}$ in that jet goes, but the same isn't true for the $1 \mathrm{D}$ distributions.

\subsection{Linear Radial Geometric Moment: Girth, Width, or Jet Broadening}

The linear radial moment, or girth, is a special case of a generic radial moment with $f(r)=r$. For discrete constituents, it is defined as

$$
\text { Girth : } \quad g=\sum_{i \in \text { jet }} \frac{p_{T}^{i}}{p_{T}^{j e t}} r_{i}
$$

The girth distribution is shown in Figure 13.

ATLAS calls this variable width. This is a hadron-collider version of a popular LEP variable called jet broadening. Jet Broadening, as measured at ALEPH [8] and OPAL [9], leads to distributions very similar to the linear moment, simply because the small-angle approximation of $k_{T} \approx p_{T} r$ is valid. At LEP, jet broadening was given by

$$
B_{\text {jet }}=\frac{\sum_{i}\left|\vec{p}_{i} \times \hat{n}_{\mathrm{jet}}\right|}{\sum_{i}\left|\vec{p}_{i}\right|}=\frac{\sum_{i}\left|\vec{k}_{T} i\right|}{\sum_{i}\left|\vec{p}_{i}\right|}
$$



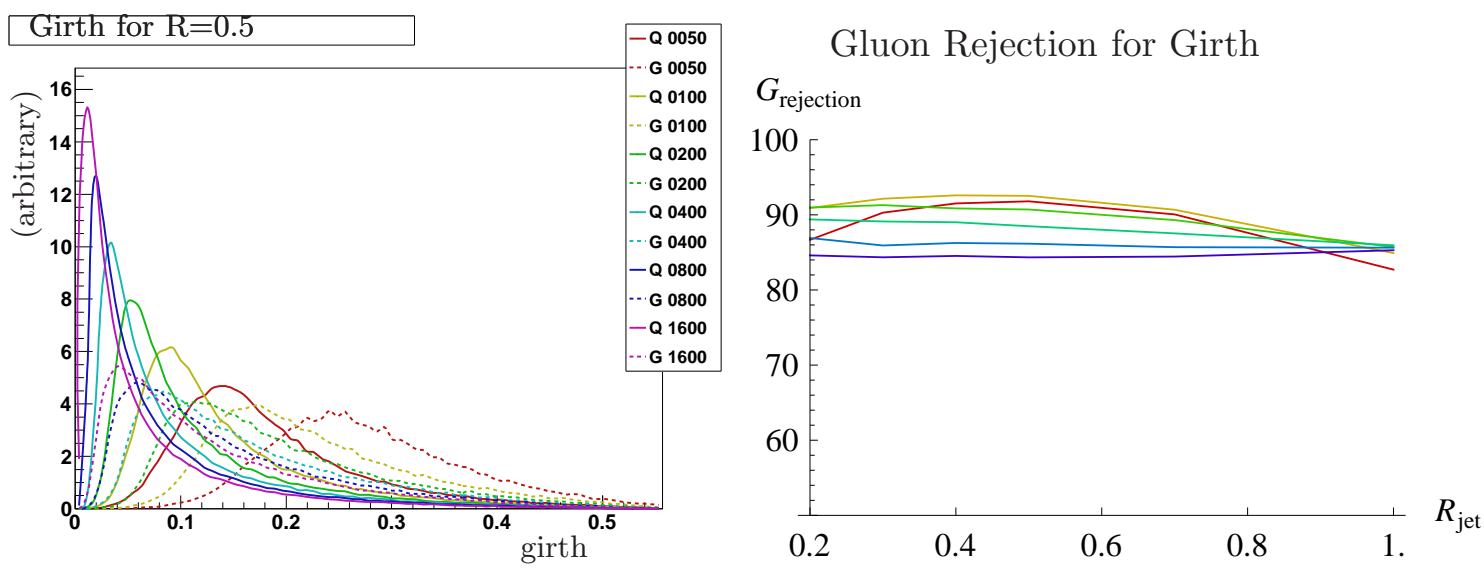

Figure 13. Girth (also called width, or the linear radial moment) for PyтнIA 8 anti- $k_{T}$ particle jets. Left: Different jet $p_{T} \mathrm{~s}$ for $\mathrm{R}=0.5$ jets, normalized to equal area. Right: Gluon rejection scores as a function of jet size for different $p_{T}$ samples. Red is $50 \mathrm{GeV}$, yellow is $100 \mathrm{GeV}$, and so on, doubling through the spectrum until purple at $1600 \mathrm{GeV}$. For the lower $p_{T}$ samples, the best gluon rejection occurs around $\mathrm{R}=0.5$.

We examined higher-power radial moments, and found them less useful for quark/gluon discrimination. CMS [37] has examined the second radial moment. For small narrow, nearly transverse jets, the second moment is equivalent to the jet mass. Higher-powered moments have the disadvantage of being most sensitive to the edges of the jet, where the most contamination lies.

On the other hand, we have found that a very good discriminator uses a lower power, specifically the square root of the distance:

$$
M_{1 / 2}=\sum_{i \in \text { jet }} \frac{p_{T}^{i}}{p_{T}^{j e t}} \sqrt{r_{i}} .
$$

\subsection{Jet Angularities}

Jet Angularities are also radial moments, but their "radial distances" are rescaled into the angular coordinates appropriate for $e^{+} e^{-}$event shapes. They are defined by [38] as

$$
\text { Jet Angularities : } \quad A_{a}=\sum_{i \in \mathrm{jet}} E_{i} f_{a}(\tilde{\theta}),
$$

with

$$
f_{a}(\tilde{\theta})=\sin ^{a} \tilde{\theta}(1-\cos \tilde{\theta})^{1-a} \quad \text { and } \quad \tilde{\theta}=\frac{\pi\left|r_{i}\right|}{2 R}
$$

for $a<2$. The kernel function $f_{a}(\theta)$ is inspired by full event-shape angularities [39], but squished so that the edge of a jet at $\left|r_{i}\right|=R$ is mapped to $\pi / 2$. Profiles for different choices of the $a$ parameter are shown in Figure 14. Note that the energies $E_{i}$ are used in the definition, instead of the $p_{T}$ s popular with hadron colliders. Also, angularities are often normalized by 


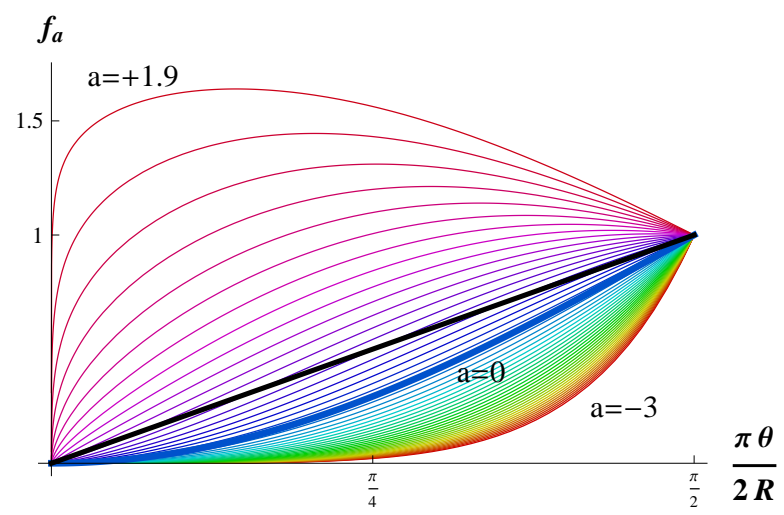

Figure 14. Profiles $f_{a}(\tilde{\theta})$ for different choices of the angularity $a$ parameter spaced at 0.1 intervals (in rainbow) and linear radial-moment "girth" (in black). These profile shapes have nothing to do with the shapes of the distributions resulting from integrating these moments over jets and forming a histogram of the results.
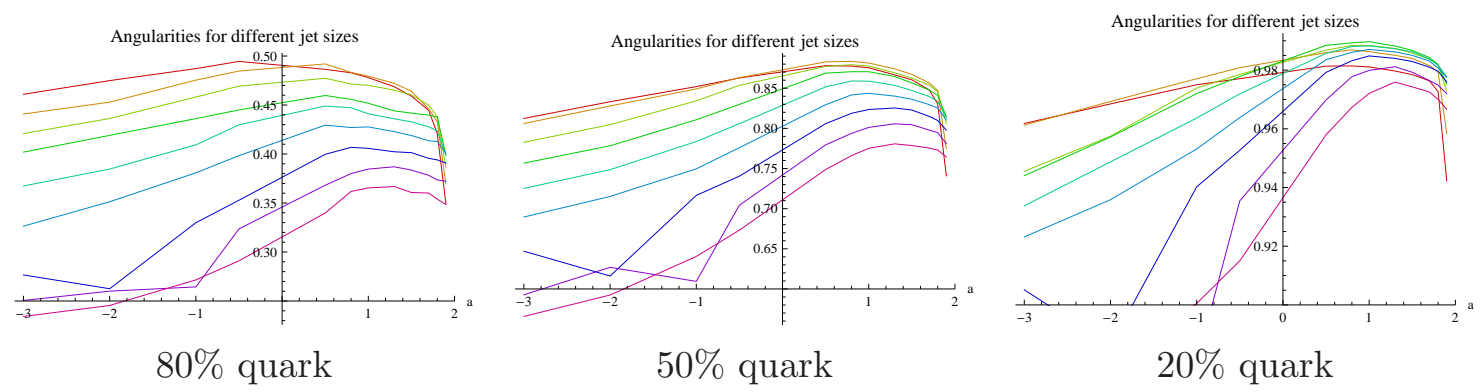

Figure 15. Gluon rejection power for angularities as a function of angularity parameter $a$. Each line represents growing PyтHIA8 particle jet size from $R=0.2$ in red up to $R=1.0$ in purple. Here the scores for all $p_{T} \mathrm{~s}$ are averaged. The best angularities perform slightly better than masses, but worse than track and subjet counts.

the jet mass, but this is not the most useful for our purposes. A given angularity has two parameters $\left(R_{\text {jet }}\right.$ and $\left.a\right)$ in addition to any discrete choices like normalization (none, jet mass, jet $p_{T}$, jet $E$ ) or angle used $(\tilde{\theta}$ as defined, or geometric $\theta$.) Gluon rejections for different choices of $a$ are shown in Figure 15.

\subsection{Optimal Kernel for Radial Moment}

Rather than sticking to powers of $r$, sines and cosines (like angularity), or another orthonormal basis, we looked for the kernel $f(r)$ that gives the best discrimination power between quarks and gluons for each $p_{T}$. Because the goal is to find the best function, the optimization problem is technically infinite dimensional. But through reasonable smoothness criteria, it can be reduced to adjusting a few control-points of a spline or coefficients of an orthonormal basis. Since adding a constant doesn't change the discrimination power, we chose our kernels 
Optimal Kernel $(\log r)$

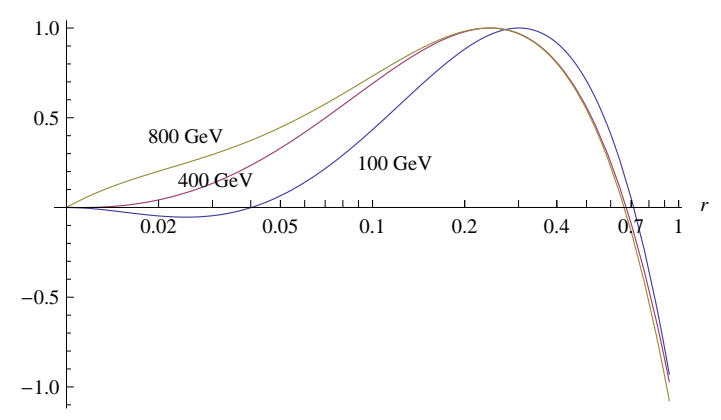

Optimal Kernel (linear r)

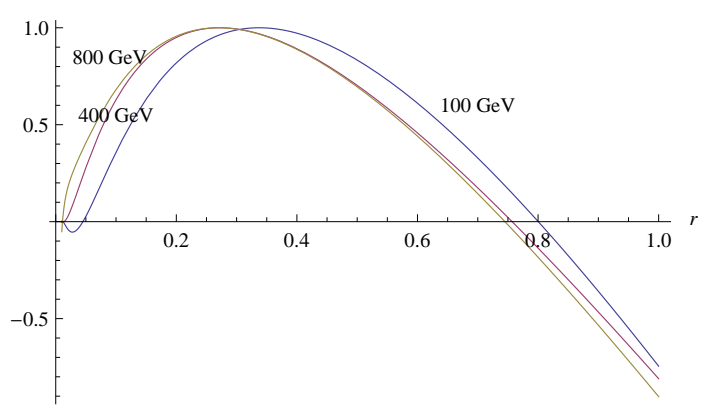

Figure 16. Profiles for the optimal kernels found for various jet sizes. Kernels for the higher- $p_{T}$ jets give a higher weight to $p_{T}$ near the jet axis.

to have $f(0)=0$. This means that the energy deposits near the crowded and noisy jet center count least. Multiplying by a constant (even a negative one) also does not affect discrimination power, so we normalized our trial profiles so their maximum value was +1 . We evaluated the ROC curve at three different quark efficiencies, $20 \%, 50 \%$, and $80 \%$.

The best kernels we found had rejection scores that were not significantly higher than those for girth (equation 8.3) or the square-root profile (equation 8.5). For this reason, we won't go into much more detail. Some general trends did appear. By construction, all kernels started out at zero at the center of the jet and rose to +1 at some distance away. In the best kernels, this happened around $r=0.4$ for low- $p T$ jets, 0.3 for $100 \mathrm{GeV}$ jets and 0.24 for 400 and $800 \mathrm{GeV}$ jets. Beyond this, it mattered less what happened, but the best kernels did fall toward the edge of the jet. Examples of such kernels are shown in Figure 16.

\subsection{N-subjettiness}

N-subjettiness [40] is a family of jet shapes that attempt to characterize the degree to which a jet has exactly $N$ subjets. $N$ is one of the input parameters, and is commonly taken to be 1 , 2 or 3 . $N$-subjettiness finds exactly $N$ axes within the jet and associates each particle or $p_{T}$ deposit to the nearest axis. These are the $N$ subjets. The $N$-subjettiness score $\tau_{N}$ is sum of $p_{T}$-weighted radial moments for each subjet. In this moment, each bit of $p_{T}$ is multiplied by its distance to the subjet axis $\Delta R$ raised to a power $\beta$, which must be positive. Specifically, this is

$$
\tau_{N, \beta}=\frac{1}{d_{0}} \sum_{J=0}^{N} \sum_{k \in \text { subjet }_{J}} p_{T, k}\left(\Delta R_{J, k}\right)^{\beta},
$$

where $d_{0}$ is a normalization involving the jet size $R_{0}$ to keep $\tau_{N, \beta}$ between zero and one:

$$
d_{0}=R_{0}^{\beta} \sum_{k \in \text { jet }} p_{T, k}
$$

There are three parameters: $N$, the exponent $\beta$, and the method of choosing axes. A simple way of choosing $N$ axes is to undo a $k_{T}$ or Cambridge-Aachen clustering exactly $N$ steps. A 
more effective method is to choose the axes that minimize the score $\tau$. It's clear from the definition that the more a jet looks like it contains exactly $N$ well-separated and individually well-collimated subjets, the lower the $\tau$ score is.

In the gluon-rejection plots in Figure 17, only charged tracks were included at ATLAS's request. If a jet contains only $N$ charged tracks (with $p_{T}>1 \mathrm{GeV}$ here), the axes will coincide with these tracks and the score will be zero. It will also be zero if there are fewer than $N$ tracks. This explains the excess in the zero-bin of some $N$-subjettiness distributions (not shown). $N$-subjettiness is also appealing because it can be calculated accurately in QCD, at least in some contexts [41].

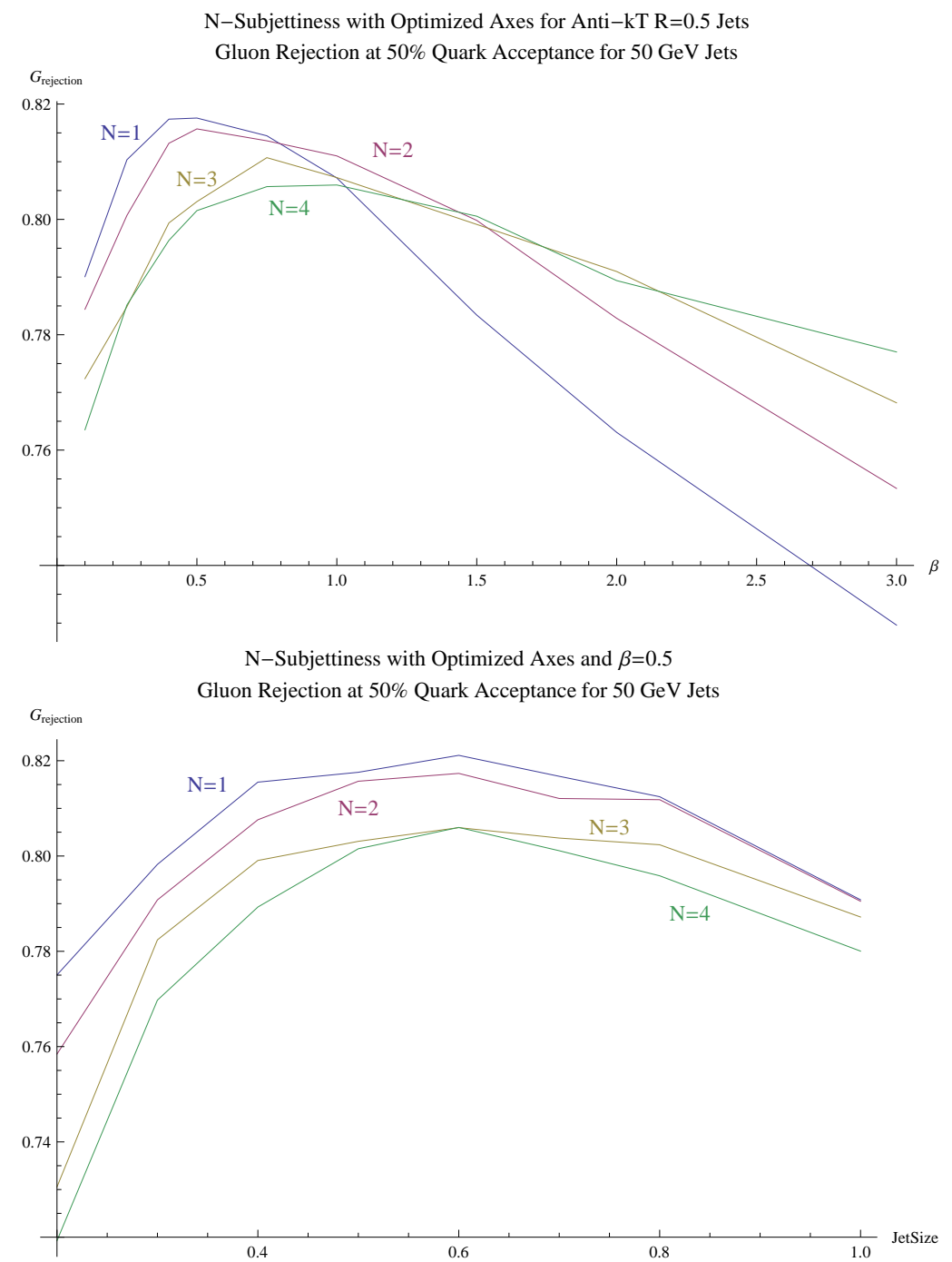

Figure 17. N-subjettiness gluon rejection as the parameters $N, \beta$, and jet-size are varied. This is for $50 \mathrm{GeV}$ particle jets simulated in Herwig ++ . The best $\beta$ s are between $1 / 2$ and 1 . As usual, the best jet sizes are between 0.5 and 0.7. These trends hold for PYтнIA 8 and for higher $p_{T}$ jets. 


\subsection{Two-Point Moment}

A two-point moment is a sum over every pair of constituents (energy deposits or tracks). It is a sum of the product of $p_{T} \mathrm{~s}$ of each pair, times their separation $\Delta R$ raised to a power $\beta$. It is normalized by the jet $p_{T}^{2}$ to make it dimensionless and less sensitive to the jet $p_{T}$ itself.

$$
T_{\beta}=\frac{1}{\left(p_{T}^{\text {jet }}\right)^{2}} \sum_{i \in \text { jet }} \sum_{j \in \text { jet }} p_{T}^{i} p_{T}^{j} \Delta R^{\beta}
$$

It is a moment of the two-point function, which would be a function of $\Delta R$. As long as $\beta>0$, this is IRC safe. This is meant to capture an average separation between constituents. In Figure 18, the gluon rejection is shown as a function of the jet size for different values of $\beta$.

Two-Point Moment for different $\beta$ 's Gluon Rejection at 50\% Quark Acceptance for $50 \mathrm{GeV}$ Jets

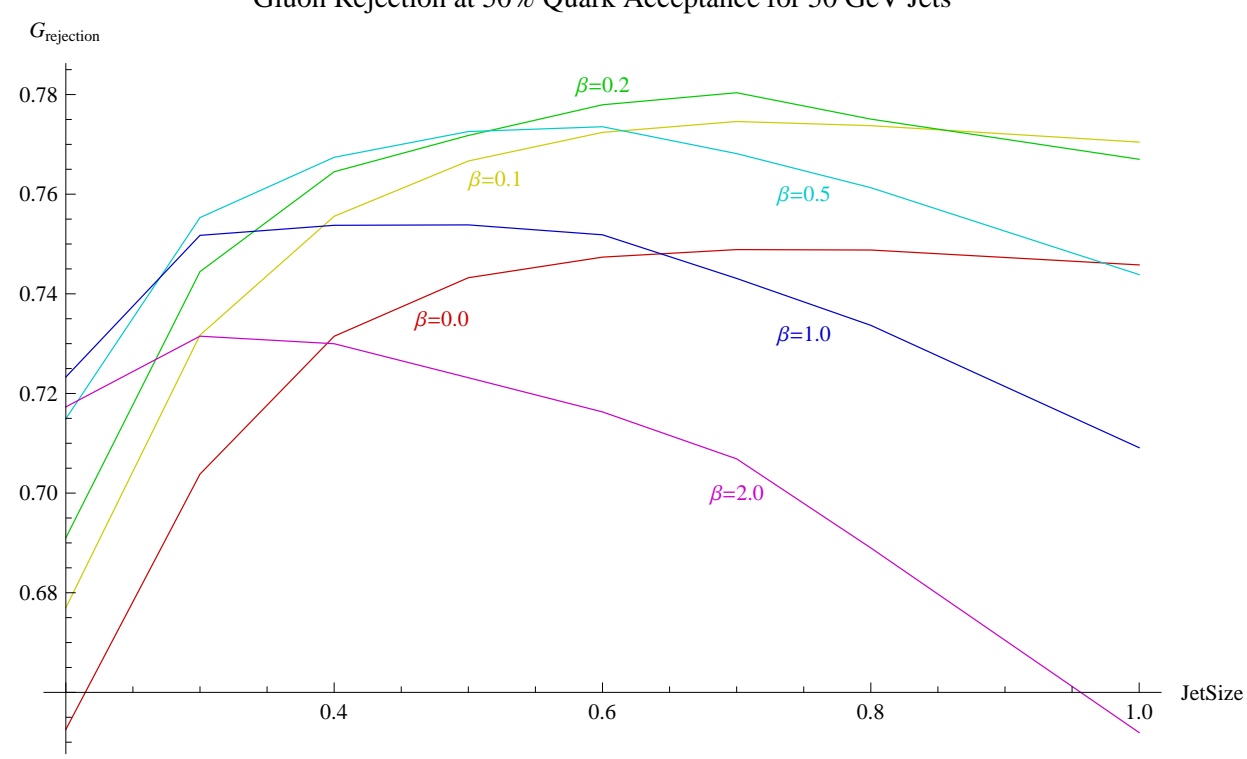

Figure 18. Two-Point Moment gluon rejection as the jet size and $\beta$ parameter are varied. This is for $50 \mathrm{GeV}$ charged-track jets simulated in HeRwIG ++ . The best $\beta$ 's are small, around $1 / 4$. As usual, the best jet sizes are between 0.5 and 0.7. These trends hold for all-particle jets, Pyтнia8, and for higher $p_{T}$. 


\subsection{Two-Dimensional Geometric Moments}

The radial moments above ignored how the $p_{T}$ was distributed around the jet axis. Motivated by the moment-of-inertia and covariance tensors, a second order $2 \mathrm{D}$ geometric moment tensor can be formed as shown in Figure 19. Combinations of its eigenvalues and eigenvectors (like Planar Flow) have been used used to distinguish boosted objects.

None of these variables turn out not be particularly useful for quark/gluon discrimination, so no distributions are shown here. Whether a quark emits a gluon or a gluon splits, the the 2-body kinematics are similar. Since it's this leading emission that dominates the subsequent shower, it is understandable that these shapes might not differ significantly between quarks and gluons.

$$
\text { Covariance Tensor: } \mathbf{C}=\sum_{i \in \mathrm{jet}} \frac{p_{T}^{i}}{p_{T}^{j e t}}\left(\begin{array}{cc}
\Delta \eta_{i} \Delta \eta_{i} \Delta \eta_{i} \Delta \phi_{i} \\
\Delta \phi_{i} \Delta \eta_{i} \Delta \phi_{i} \Delta \phi_{i}
\end{array}\right)
$$

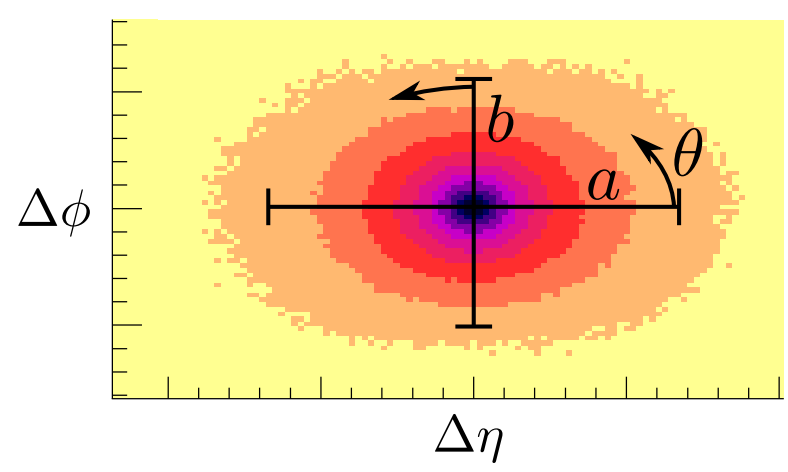

\section{Combination of Eigenvalues}

Eigenvalues: $a>b$

$$
\text { Quadratic Moment: } g=\sqrt{a^{2}+b^{2}}
$$

Determinant: $\operatorname{det}=a \cdot b$

Ratio: $\rho=b / a$

Eccentricity: $\quad \epsilon=\sqrt{a^{2}-b^{2}}$

Planar Flow: $\quad p f=\frac{4 a b}{(a+b)^{2}}$

Orientation: $\theta$

Figure 19. The Covariance Tensor and its eigenvalues and eigenvectors. 


\subsection{Pull}

In figure 20, we show accumulated $p_{T}$ for the same quark and gluon parton showered millions of times. In the large $N_{C}$ approximation where these concepts apply, quarks have a single color connection and gluons have one color and one anti-color connection. In this particular event, the quark was color-connected with the beam remnant that went off to the left toward $\eta=-\infty$. The gluon was connected to both outgoing beams.

Pull tries to quantify the color connections. It was introduced in Ref. [36], and then immediately used in the $\mathrm{D} \varnothing$ search for $Z H$ with $Z \rightarrow \nu \bar{\nu}$ [42]. The pull vector of a jet is designed to point toward the jet or beam that its color-connected to. The pull vector is a $p_{T}$ weighted moment that tends to point toward the color-connected partner of the jet's initiating quark. If the jet was initiated by a gluon, it is color-connected two two different places, so we might expect less pull. The pull vector is defined as

$$
\text { Pull Vector } \vec{t}=\sum_{i \in \text { jet }} \frac{p_{T}^{i}\left|r_{i}\right|}{p_{T}^{j e t}} \vec{r}_{i} \quad \text { where } \vec{r}_{i} \equiv\left(y_{i}-y_{\text {jet }}, \phi_{i}-\phi_{\text {jet }}\right) \text {. }
$$

If the factor of $\left|r_{i}\right|$ were removed, this would be the jet's $p_{T}$-weighted centroid. Unlike other moments, the pull vector is explicitly designed to not be rotationally invariant. The most effective way to use the pull vector in the Higgs study was to calculate a pull angle, which is the angle between the pull vector and the direction where it 'should' point if the jet was color-connected to some other object. We did not find pull angle very useful in distinguishing quarks from gluons.

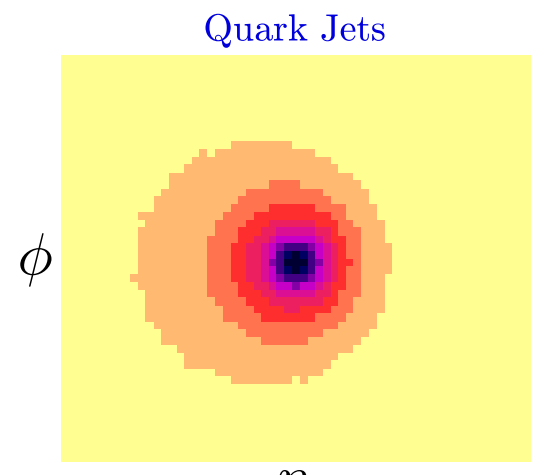

$\eta$

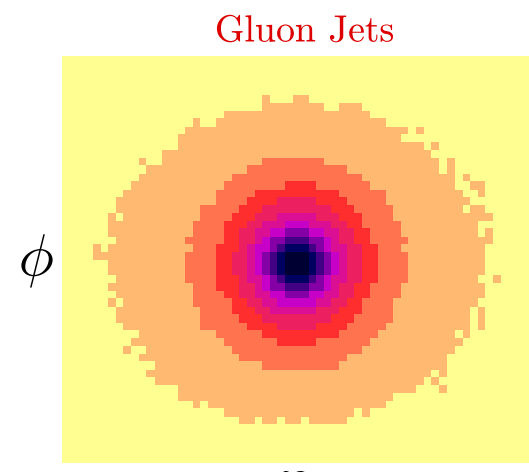

$\eta$

Figure 20. Distribution of radiation in quark and gluon jets accumulated over 3 million back-to-back $100 \mathrm{GeV}$ dijet events with fixed parton kinematics. The color shows the average showered $p_{T}$ density in $(\eta, \phi)$ for an ensemble of events with fixed parton-level kinematics. (Contours are stepped in factors of two, which somewhat obscures the nearly identical jet $p_{T}$ in both cases.) 


\section{Combining Variables}

A multivariate tagger can make the best use of several variables at the same time. In Figure 21, the $2 \mathrm{D}$ distributions of a good pair of variables is plotted for the quark and gluon samples. To find the best cut contours, one method is to combining these histograms into a likelihood histogram. This is done bin-by-bin by reading the values of the quark and gluon histograms and computing $q /(q+g)$. If particular values are measured for each of the two variables, this likelihood is proportional to the probability that it is a quark jet. The constant of proportionality will depend on the prior distribution of quarks and gluons in your sample via Bayes' Theorem, but does not affect the contours.

A cut on on this likelihood score corresponds to a cut along some contour in the $2 \mathrm{D}$ plane. Each such cut gives some efficiency for keeping quark jets and some other efficiency for rejecting gluon jets. Cutting on the likelihood is optimal in the sense that it maximizes gluon rejection for every given quark acceptance [25]. Some ways of visualizing the effects of cuts and multivariate improvements were discussed in [43, 44].

To populate a $2 \mathrm{D}$ histograms such that each bin has a statistically meaningful number is difficult without an enormous number of events. For more than 2 variables, it is practically impossible to populate the higher-dimensional histograms with any accuracy. For example, for 5 variables, even if each variable had only 10 coarse divisions, there would still be $10^{5}$ bins to populate. This is where multivariate techniques like Boosted Decision Trees are useful [25]. Using a limited number of training events, these techniques assign a score to each point. With a large enough training sample, this score is in 1-1 correspondence with the likelihood.
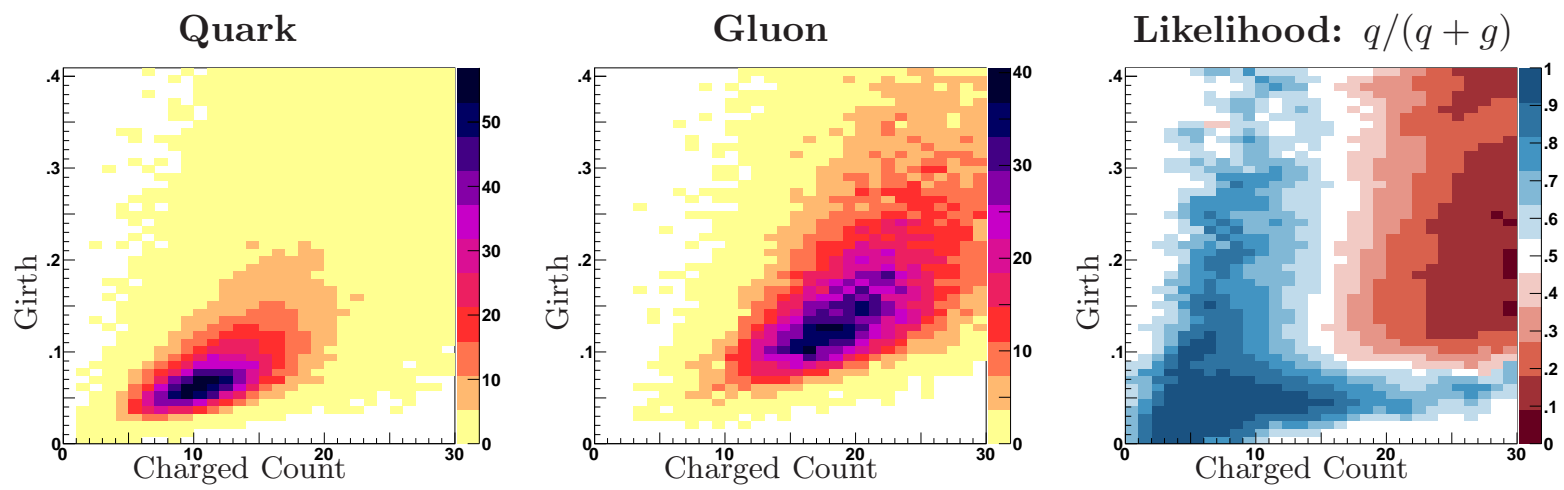

Figure 21. Combining Variables: 2D distributions are shown for a powerful pair of variables. The Likelihood can be formed by combining these histograms bin-by-bin as $q /(q+g)$, where $q$ and $g$ are the fraction of events in the appropriate bin of the quark and gluon histogram, respectively. The blue regions mean that an event with that pair of values is more likely to be quark. A cut on the likelihood correspond to a cut along one of the contours, and this can be proven to be the optimal cut for that signal efficiency. These plots are for PyтнIA8 $200 \mathrm{GeV}$ particle jets. 


\section{Comparing Variables}

A pair of variables that always performs at or near the top in our multivariate rankings is charged track count combined with girth (also known as the linear radial moment jet width). This pair was shown in Figure 21. In a computationally intensive search, we also came up with the best group of five variables, which differed for each $p_{T}$ window and ranking method (gluon rejection at $80 \%, 50 \%$, or $20 \%$.)

The ROC curves (gluon rejection versus quark acceptance) for interesting variables are shown in Figure 22 for $p_{T}=100 \mathrm{GeV}$ jets. Unlike previous curves, the rejection is plotted on a logarithmic axis. A $1 \%$ background acceptance corresponds to a background rejection of $10^{2}$. The best curve corresponds to the best group of five variables, but the best pair (charged track multiplicity \& girth) is not far behind. Simply taking the product of these two creates a single variable that does better than each individually, but only for for harder cuts (lower quark acceptances.) The good discrete observables like counting charged tracks or counting small subjets do best at high quark acceptance (and as we saw before, high jet $p_{T}$ ). The good continuous observables like girth tend to do best at lower quark acceptance and lower jet $p_{T}$. Jet mass tends to be somewhere in the middle, and $2 \mathrm{D}$ geometric moments like pull and planar flow are never particularly powerful.

The best variable depends on the desired signal acceptance operating point, which depends on the application. For example, one might try to maximize the significance $(\sigma \sim$ $S / \sqrt{B}$ ) of a small signal above a large background. An advantage of maximizing the significance is that, each operating point translates into an improvement factor (which should be greater than one if the cut is useful), independent of the initial significance. This improvement factor is also independent of integrated luminosity and the signal and background counts themselves. To see this, note that cutting on a variable changes the significance by

$$
\sigma \equiv \frac{S}{\sqrt{B}} \quad \stackrel{\text { cut }}{\rightarrow} \quad \frac{\varepsilon_{S} S}{\sqrt{\varepsilon_{B} B}}=\left(\frac{\varepsilon_{S}}{\sqrt{\varepsilon_{B}}}\right) \sigma .
$$

With a simple 1-1 transformation, a ROC curve can be turned into a significance improvement curve (SIC) [43]. Samples are shown in Figure 23 for the $100 \mathrm{GeV}$ sample.

For all variables, the cuts that optimize quark/gluon significance improvement tend to be quite harsh, leaving only $\sim 20 \%$ of the quark sample. For rare signals with few events, looser cuts might be required to see any events at all. In cases where the background to a quark jet new physics signal is not $100 \%$ gluon-jets, looser cuts end up giving the optimal significance improvement. QCD backgrounds at low $p_{T}$ are only around $80 \%$ gluon. This is discussed further in Section 12.

Gluon rejection curves for the best group of five variables for each of the $p_{T}$ samples are shown in Figure 24. There is one best group of five when optimizing rejection at $20 \%$ quark acceptance, and a different group of five when optimizing at $80 \%$. Transformations of these into Significance Improvement Curves is shown in Figure 25. Exact scores depend on whether Pythia8 or HeRwig ++ is used and whether all particles are used or just charged tracks. This is discussed further in Section 13. Results are shown at the end, in Table 1. 


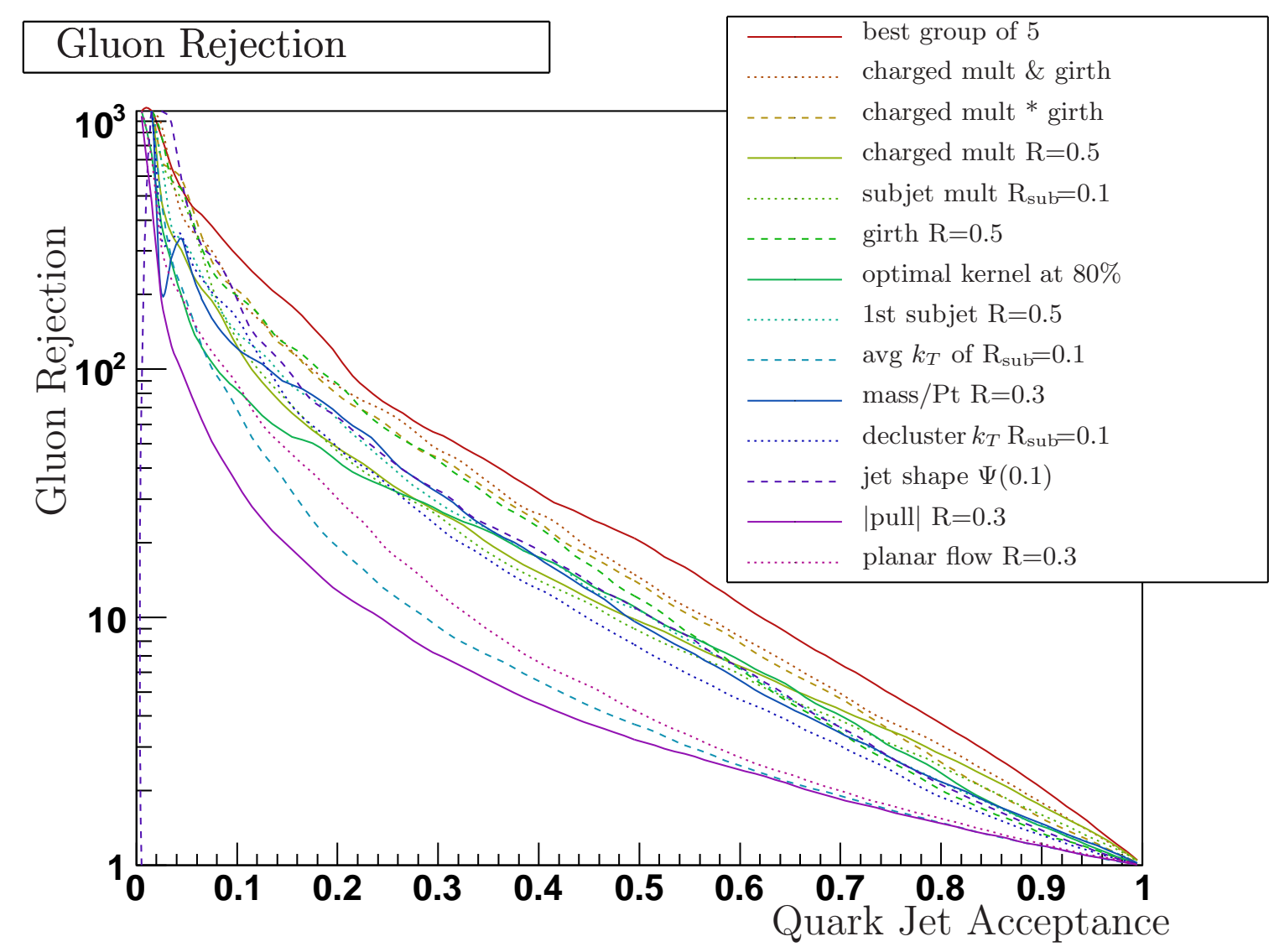

Figure 22. ROC curves for $100 \mathrm{GeV}$ Pythia 8 jets for selected variables. These curves show the background (gluon jet) rejection efficiency $\left(1 / \varepsilon_{B}\right)$ as a function of the signal (quark jet) acceptance efficiency $\left(\varepsilon_{S}\right)$. 


\section{Significance Improvement}

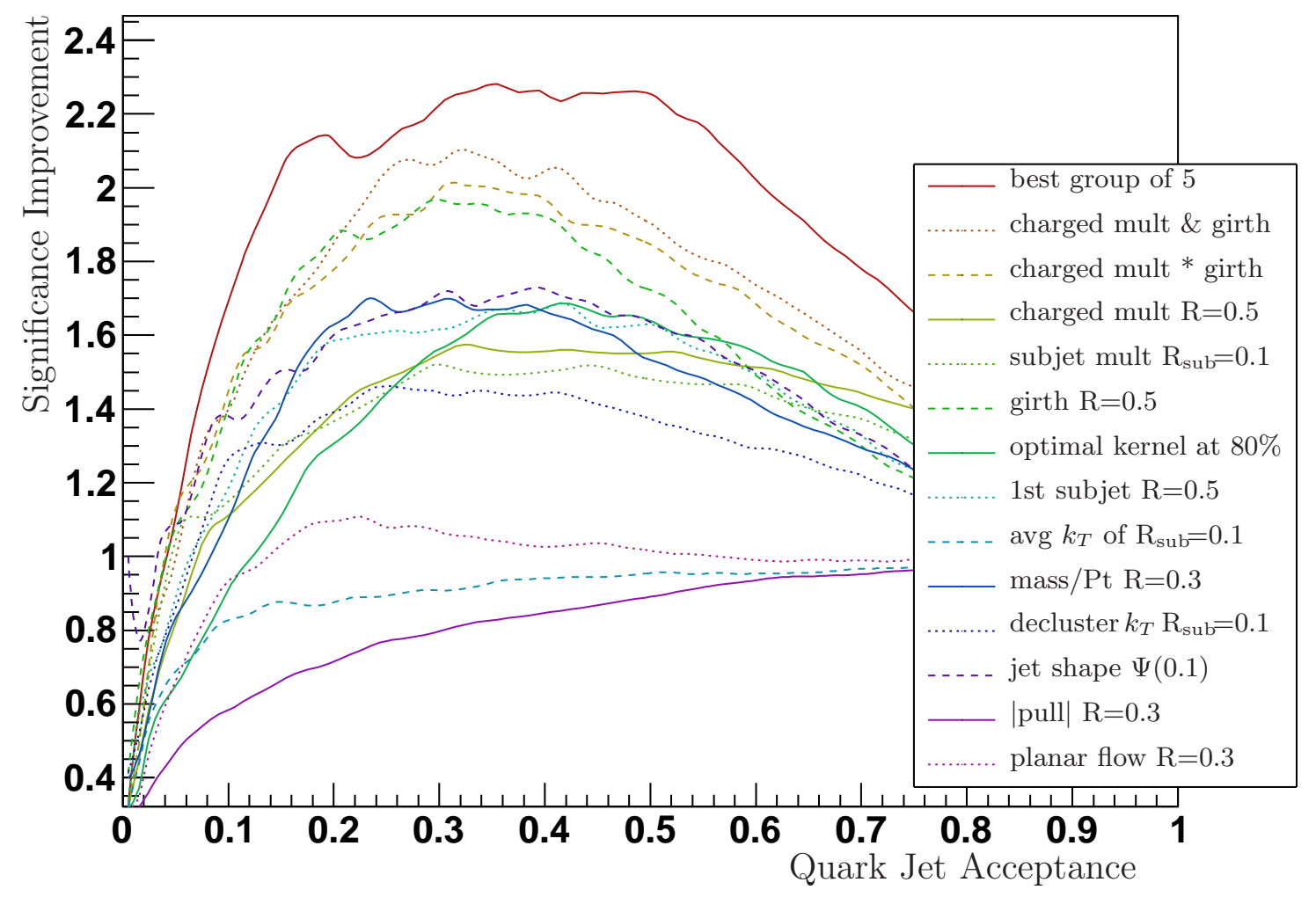

Figure 23. Significance Improvement Curves for $100 \mathrm{GeV}$ Pyтнia 8 jets for selected variables. These curves show the significance improvement $\varepsilon_{S} / \sqrt{\varepsilon_{B}}$ as a function of $\varepsilon_{S}$. 


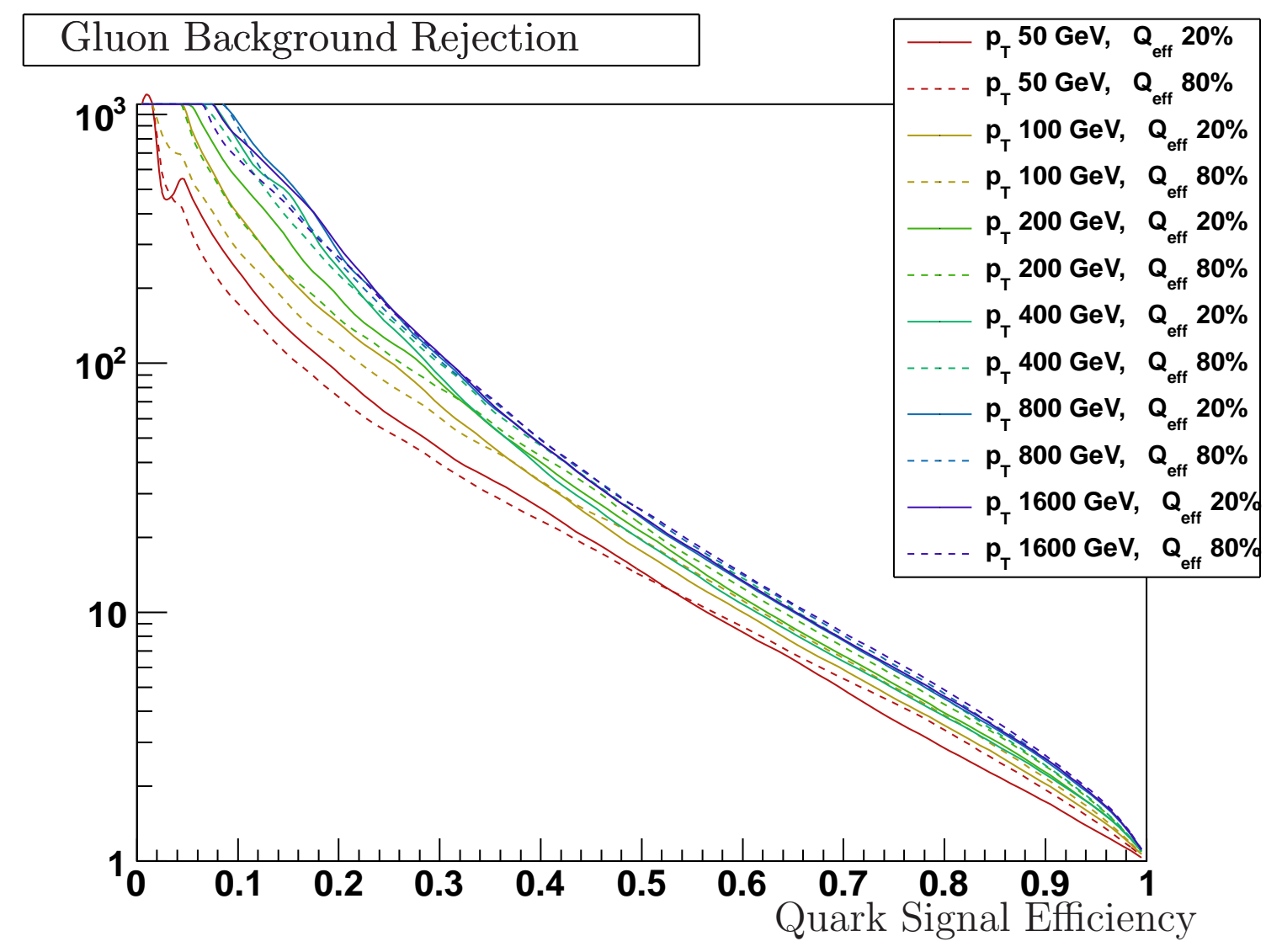

Figure 24. Gluon Background Rejection for the best groups of five PyTHIA8 variables for each $p_{T}$. The dashed lines are the groups that maximize gluon rejection at $80 \%$ quark efficiency. The solid lines maximize significance improvement, which the next figure shows happens around $\sim 20 \%$ quark efficiency. Higher $p_{T}$ jets lead to greater rejection power, mostly because the charged track count is a more powerful observable. 


\section{Significance Improvement}

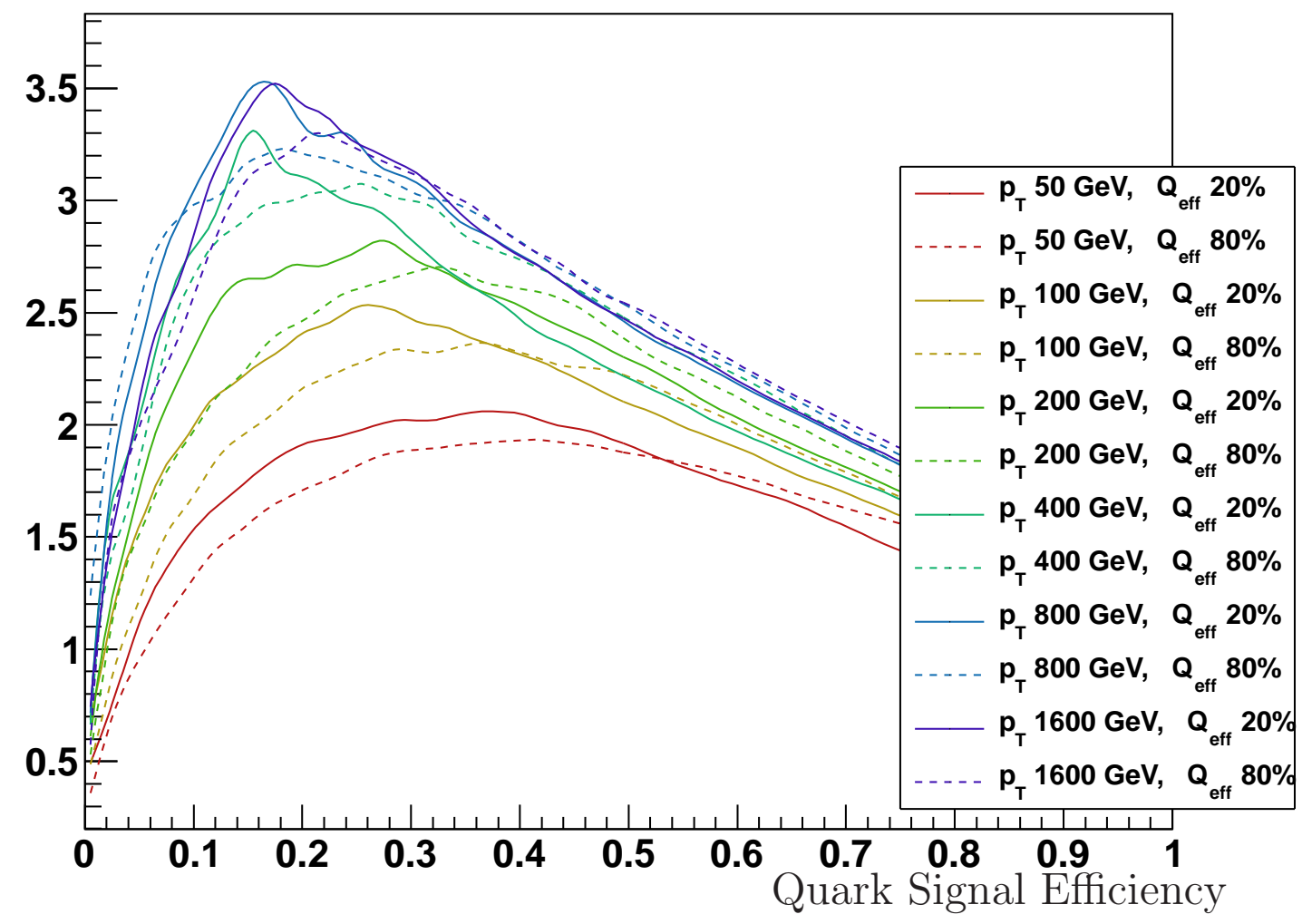

Figure 25. Significance Improvement curves for best groups of five variables. This contains the same information as the previous figure, but shows that small differences in gluon rejection at low efficiencies lead to large differences in significance improvement. 


\section{Using Impure Samples to Verify Underlying Pure Distributions}

When training a multivariate method to distinguish quarks from gluons, it would be ideal to have huge samples of pure quarks and pure gluons. The quark fractions of several samples are shown in Figure 26. For low jet $p_{T}$, none of the samples are more $90 \%$ pure. By making cuts, this can be increased at the cost of having fewer training events. For example, in $\gamma+2$ jet events, when the softer jet is close to the photon, it is very likely a quark jet. Similar cuts can be made to purify gluons from multi-jet samples. This was discussed in reference [3].

Ideally, one would like to combine information from high cross section, low-purity samples with low cross section, high-purity samples. One approach to combining information from different samples would be to first verify the jet property predictions Monte Carlo generators. If the Monte Carlo generators are sufficiently accurate, huge numbers of simulated events can then be used to train multivariate classifiers. The distributions of each jet property and correlations between them can be checked against data. For example, the jet mass distribution of a simulated 90/10 mix should match a 90\% pure sample. If two observables provide most of the discrimination power, their 2D histogram can be compared to the linear combination of pure quark and gluon jets produced by the Monte Carlo. Low statistics, high purity samples

\section{Chance EACH Jet is Quark}

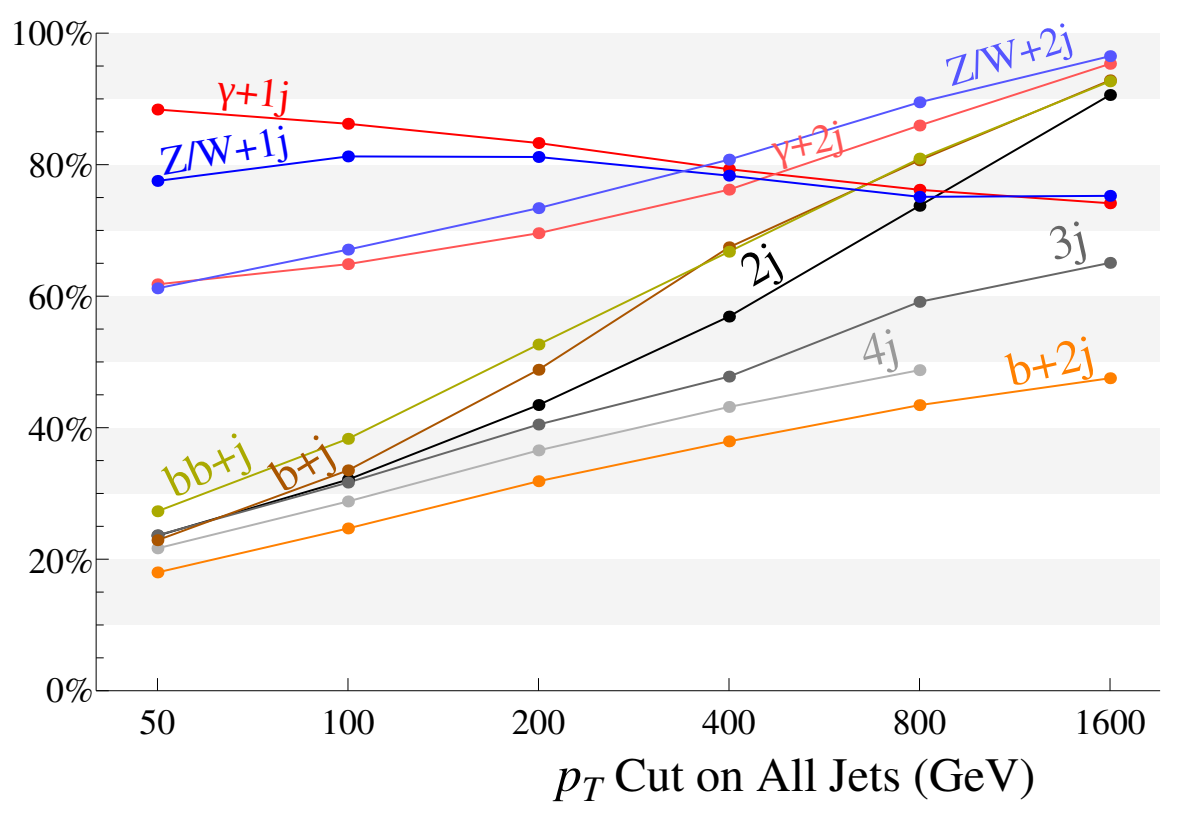

Figure 26. The chance that a given jet is a light quark jet rather than a gluon jet. (This ratio does not include bottom or charm.) The $W$ and $Z$ were nearly identical and combined on this plot, but they are slightly different from the photon, mostly due to the $\gamma$ and lepton cuts, which were each at $20 \mathrm{GeV}$. 
will constrain some combination of measurements, while high statistics mixed samples will constrain a different combination.

More generally, each jet can be assigned a likelihood of being quark or gluon based on its kinematics (nearness to a photon for example) rather than its intra-jet properties. If it passes some kinematic threshold, the jet and its likelihood can be used to train a classifier. More certain probabilities can be assigned higher weights. Although the multivariate techniques popular in high energy physics and implemented in the TMVA package [25] require a separate signal and background samples, within each sample events can be assigned different weights.

An alternative data-driven approach is to fit for the underlying pure distributions in an impure sample where the quark/gluon fractions are assumed to be known. For example, in a small window around $50 \mathrm{GeV}$, dijets with certain kinematic cuts are $23 \%$ quarks. Jets in a $\gamma+$ jet sample are $88 \%$ quarks. If the jet mass distribution is measured for these two $50 \mathrm{GeV}$ samples, the underlying quark and gluon distributions can found by solving a simple 2x2 linear equation bin-by-bin. These pure underlying quark and gluon mass distributions can then be re-weighted and compared to a sample with a different known quark/gluon fraction. If there is consistency across samples, it would justify the use of quark and gluon jet discrimination. ATLAS calls this the template method $[28,45]$. In this approach, bottom and charm jets are accounted for by trusting the Monte Carlo simulation for both their distribution and the value of their small composition fraction.

Fitting to pure or mixed samples assumes a universality to quark and gluon properties, which may only partly hold. Selection effects might induce atypical distributions, for example if harsh cuts keep only the kinematic tails of distributions. For example, jet mass might look different at very high $\eta$. One sample may be busier than another, with many other nearby jets leaking into the ones you are interested in. Jets in a training sample may have different color-connections than jets in the sample you ultimately wish to tag. Color-singlet quark pairs from a $W$ might look different than beam-connected quarks in $\gamma+$ jet. Many of these issues are not particular to quark/gluon tagging and should be kept in mind for any substructure study.

\section{Choosing the Operating Point for a Mixed Background}

The cut that most improves significance depends on the quark/gluon composition of the real signal and background. So far we have considered the signal to be only quark jets and the background to be only gluon jets. QCD has around $20 \%$ quark jets at low $p_{T}$ and more for higher $p_{T}$. Other common backgrounds were shown in Figure 26.

Once a tagger is trained, you need to pick an operating point. For example, you could pick the fraction of quark jets you are willing keep: the quark efficiency $\epsilon_{q}$. This translates into a particular cut on the observables, either directly or via a cut on a multivariate output. The ROC curve shows the gluon efficiency $\epsilon_{g}$ (the fraction of gluon jets that get past the best cut) for a given $\epsilon_{q}$. 
If the signal is not pure quark and the background is not pure gluon, a cut with these quark and gluon efficiencies will translate into signal and background efficiencies in a way that depends on: the fraction of signal made of quarks $s_{q}$, signal made of gluons $s_{g}$, background made of quarks $b_{q}$, and background made of gluons $b_{g}$. In this case,

$$
\epsilon_{s}=s_{q} \epsilon_{q}+s_{g} \epsilon_{g} \quad \text { and } \quad \epsilon_{b}=b_{q} \epsilon_{q}+b_{g} \epsilon_{g}
$$

Now suppose you start with $S$ signal events and $B$ background events. A cut with signal efficiency $\epsilon_{s}$ and background efficiency $\epsilon_{b}$ changes the statistical significance in a simple way as before:

$$
\sigma=\frac{S}{\sqrt{B}} \quad \rightarrow \quad \frac{S \epsilon_{s}}{\sqrt{B \epsilon_{b}}}=\sigma \frac{\epsilon_{s}}{\sqrt{\epsilon_{b}}}
$$

If the signal started with some significance $\sigma$, the cut will improve it by a factor $\epsilon_{s} / \sqrt{\epsilon_{b}}$, which is called the "Significance Improvement" in the plots below. It depends not only on the performance of the tagger, but on the quark/gluon makeup of your signal and background and where one chooses to operate. A ROC curve for quark/gluon discrimination $\left(\epsilon_{g}\right.$ vs $\left.\epsilon_{q}\right)$ can be easily transformed into a Significance Improvement Curve $\left(\epsilon_{s} / \sqrt{\epsilon_{b}}\right.$ vs $\left.\epsilon_{s}\right)$ using equations 12.1 and 12.2. The first plot in Figure 27, several such curves are shown for signals that are $100 \%$ quark, but backgrounds that are mixed. The curve labeled $0 \%$ has a background that is purely gluon jets and corresponds to the curves shown previously. Even when the background has only $10 \%$ quark jets, the maximum achievable significance improvement has fallen quite a bit. The second plot in this figure shows those maxima as a continuous function of the background's quark fraction. A typical low- $p_{T}$ QCD background has $15 \%$ quarks and is indicated. Clearly the tagger will be most useful when the signal and background are both quite pure.

The same analysis can be performed for the published $B$-tagger ROC curves. Generic low$p_{T}$ QCD backgrounds have around $2 \% B$-jets. For this value, the significance improvement peaks at around $60 \% \mathrm{~B}$-acceptance. This is the typical operating point of these taggers. 

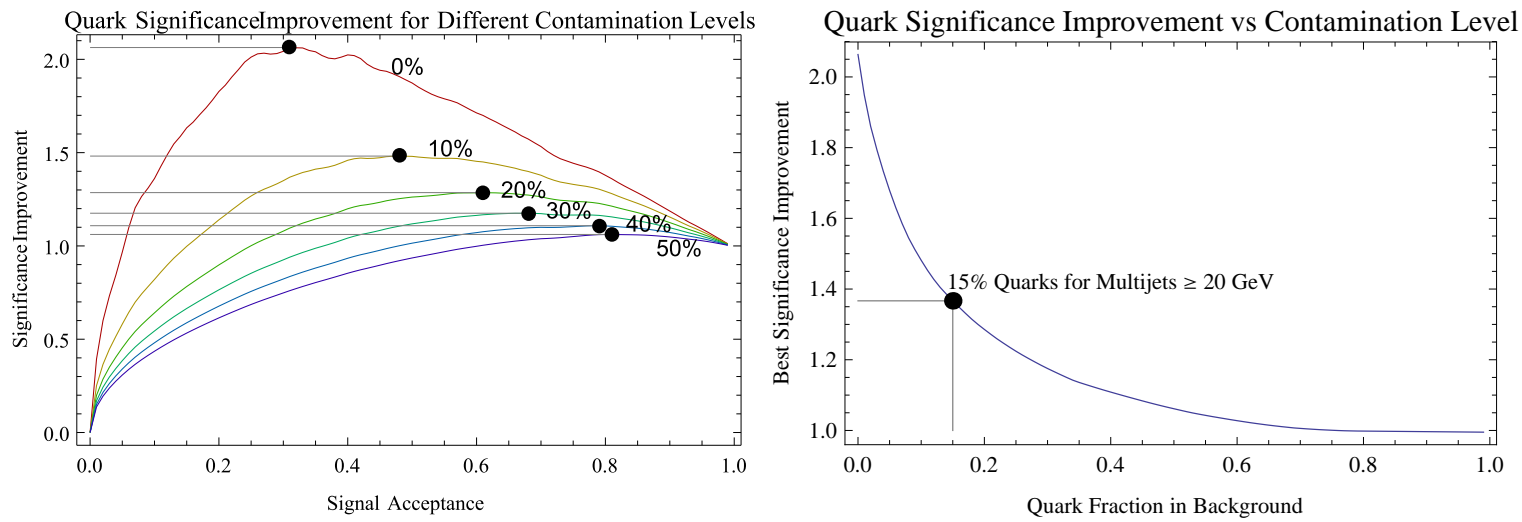

Figure 27. On the left is an illustration of how the significance improvement curve changes when the background is not pure gluon jets, but contains the indicated fraction of quark jets. The points show the maximum significance improvement. On the right, these maxima are plotted a function of the quark fraction in the background. The QCD background for jets with $p_{T} \geq 20 \mathrm{GeV}$ is approximately $15 \%$ quark. For higher $p_{T}$, the QCD quark fraction goes up and the maximum significance decreases. 


\section{Comparing HERWIG++ to PYTHIA8}

Recently, ATLAS presented some quark/gluon measurements [45] of charged track count and linear radial moment, which they call jet width. They compared their data to HeRwiG ++ and to two Pythia8 tunes. They found that both simulations described the quark jet properties better than gluon jet properties.

Our simulation, shown in Figure 28, indicates that various simulations agree with each other for quark jets. We find that the distributions for gluon jets in PYTHIA 8.165 are consistently farther away from the quark distributions than they are in HeRwIG ++ 2.5.2. ATLAS found that HERWIG ++ agreed with data better for charged tracks, but PYTHIA8 agreed better for width. In both the data and HeRwiG ++, quarks and gluons look more similar to each other than they do in Pyтнia8 (which was used for most of the plots in this paper). Gluon rejection is consistently around 10\% worse for HERWIG + + than for PyTHIA8. As a function of things like jet-size or radial moment power, or the number of subjets, the difference between HeRWIG++ and PythiA8 is just an overall shift. This means that all of the single-variable trends described in the bulk of this paper still hold. We find less advantage in combining variables with multivariate techniques for jets simulated using HERWIG ++ than with PyTHIA8.

ATLAS also found that various jet grooming techniques not only helped with pileup, but the groomed jet mass was better simulated than the ungroomed mass. To reject pileup, ATLAS also used only the charged tracks, thus ignoring their calorimeter for everything except the overall jet $p_{T}$. To account for this, in our comparison of PyTHIA 8 to HeRwiG ++ , we use only charged tracks. A summary and comparison of various simulations using all and just charged tracks is shown in Table 1.

Combining variables sometimes helps, but mostly for harder jets. At 50\% quark efficiency, combining radial moment with track count gives an additional $0.4 \%$ to $1.9 \%$ gluon rejection, depending on jet $p_{T}$ and generator. For HERWIG++, this improvement increases with jet $p_{T}$, but for Pythia8, it reaches its maximum at $200 \mathrm{GeV}$. These small shifts should be compared to gluon efficiency, not gluon rejection (one minus efficiency). For PythiA8 $200 \mathrm{GeV}$ jets, going from $9 \%$ efficiency down to $7 \%$ efficiency has a sizable effect on significance improvement. It directly translates into a $20 \%$ better $S / B$. But HeRwIG's efficiency here starts at the higher $19 \%$ and only goes down to $17.3 \%$ - a more modest $9 \%$ improvement in $S / B$. The improvements for 50 and $100 \mathrm{GeV}$ jets are smaller than for $200 \mathrm{GeV}$ jets. 
Charged Track Count $\left(n_{\text {trk }}\right)$

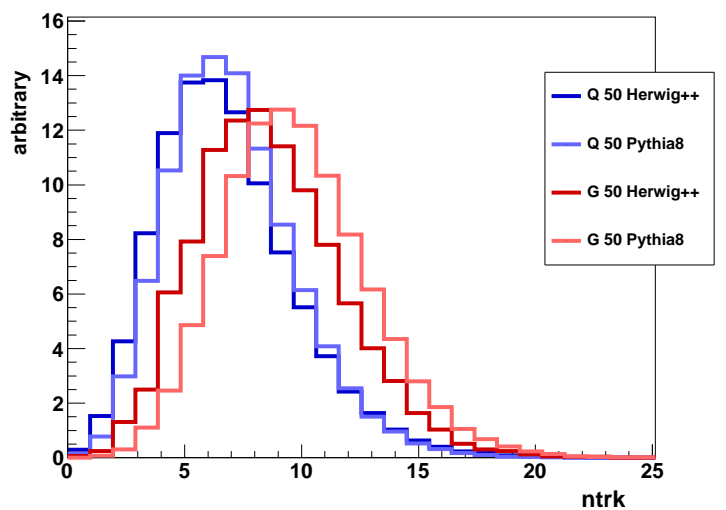

$\operatorname{mass} / p_{T}$

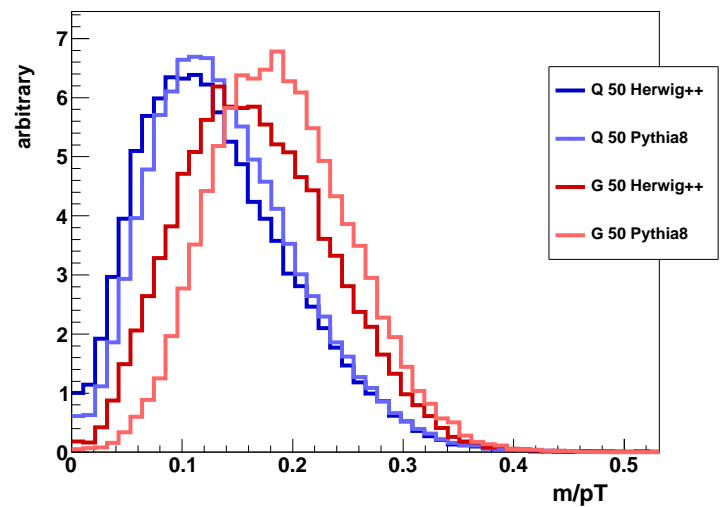

Linear Radial Moment (jet width)

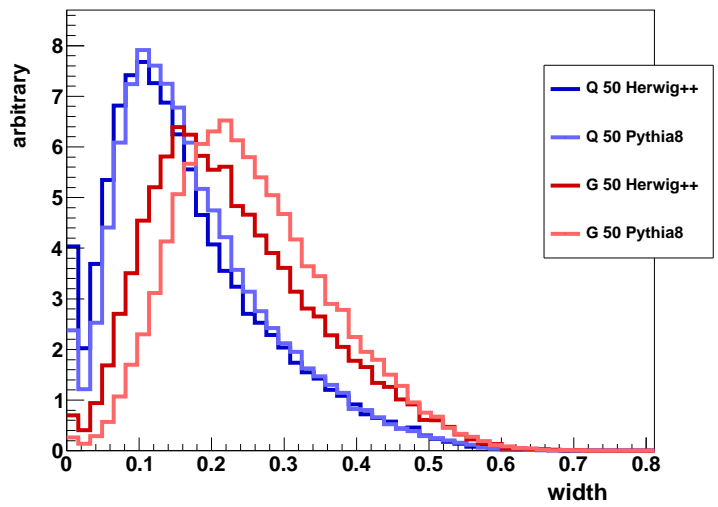

1-subjettiness, optimized axes $\beta=1 / 4$

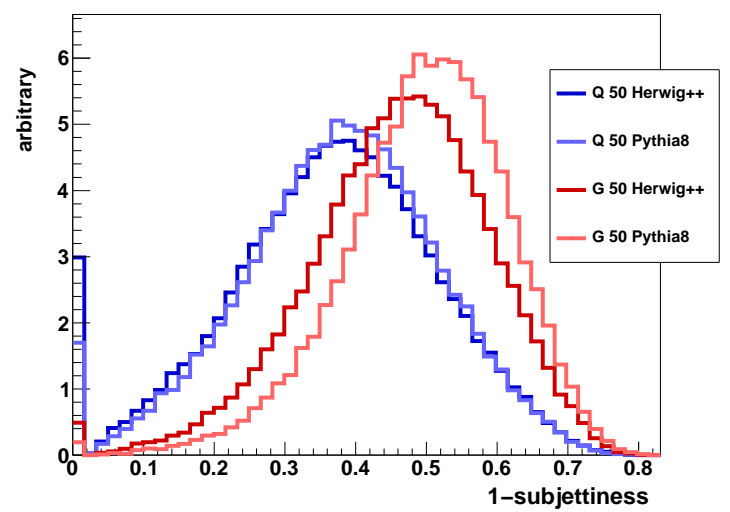

Figure 28. Distributions of charged track count and linear radial moment (here calculated using only the charged tracks within the jet) for $50 \mathrm{GeV}$ jets. Quark samples are blue and Gluon samples red. Pythia 8.165 is the lighter shade and HeRwig ++2.5 .2 is in the darker shade. 


\section{Conclusions}

In this paper, we have performed a comprehensive Monte-Carlo-based multivariate study of how quark and gluons can be distinguished on an event-by-event basis. We considered thousands of variables, which can generally be classified in two broad classes: discrete variables that count the number of tracks in a jet, and continuous variables, such as jet shapes. A general conclusion is that discrete variables help more at high jet $p_{T}$ and high quark efficiency (loose cuts), whereas continuous variables help more at lower jet $p_{T}$ and lower quark efficiency (tight cuts). Overall discrimination also works better at higher $p_{T}$.

We find, not surprisingly, that the more information that is used, the better the discrimination: counting smaller subjets work better than larger subjets; using all particles to calculate jet moments rather than just charged tracks works better. For charged track count, even using larger $R=0.7$ jets helps. The best two-variable discriminant often involves one variable from each class. Including three or more variables in a multivariate discriminant shows only small improvements over two variables. The marginal improvement of combining variables is also larger at high $p_{T}$ than at low $p_{T}$.

We have found that there is a consistent difference in how variables perform when Pythia8 or Herwig ++ is used for the simulation. Pythia8 simulations show more differences between quarks and gluons, but unfortunately, early studies with data indicate that HeRwIG ++ more accurately predicts quark/gluon discrimination power. We find that single variables can reject about $90 \%$ of gluon jets, keeping $50 \%$ of quark jets if PYTHIA 8 can be trusted, but only around $80 \%$ of gluon jets with HeRwiG ++ . Some quantitative results are shown in Table 1. Since there are significant differences between different event generators, one use of quark and gluon tagging would be to tune these generators to match data more accurately. Such improved tunings could have important implications for a number of substructure based analyses.

In conclusion, quark/gluon discrimination is difficult, but not impossible. $85 \%$ gluon rejection at $50 \%$ quark acceptance seems feasible, however further input from experiment is needed.

\section{Acknowledgments}

The authors would like to thank D. Mateos, D. Miller, A. Schwartzman, M. Silva and M. Swiatlowski for useful discussions. This work was supported in part by the Department of Energy under grant DE-SC003916. Computations for this paper were performed on the Odyssey cluster supported by the FAS Research Computing Group at Harvard University. 


\begin{tabular}{|l|c|c|c|c||c|c|c|c|}
\hline Gluon Efficiency \% at & \multicolumn{3}{|c||}{ 50 GeV } & \multicolumn{4}{c|}{ 200 GeV } \\
50\% Quark Acceptance & \multicolumn{2}{|c|}{ Particles } & \multicolumn{2}{|c|}{ Tracks } & \multicolumn{2}{|c|}{ Particles } & \multicolumn{2}{c|}{ Tracks } \\
& P8 & H++ & P8 & H++ & P8 & H++ & P8 & H++ \\
\hline 2-Point Moment $\beta=1 / 5$ & $8.7^{*}$ & $17.8^{*}$ & $13.7^{*}$ & $22.8^{*}$ & 8.3 & 15.9 & 13.2 & 19.6 \\
1-Subjettiness $\beta=1 / 2$ & 9.3 & 18.5 & 14.2 & 22.9 & 7.6 & 16.2 & 12.3 & 19.4 \\
2-Subjettiness $\beta=1 / 2$ & 9.2 & 18.6 & 13.9 & 23.6 & 6.8 & $15.7^{*}$ & 9.8 & $18.7^{*}$ \\
3-Subjettiness $\beta=1$ & 9.1 & 19.3 & 14.6 & 24.4 & $5.9^{*}$ & 16.7 & $8.6^{*}$ & 19.5 \\
Radial Moment $\beta=1($ Girth $)$ & 10.3 & 20.5 & 16.1 & 24.9 & 11.2 & 18.9 & 15.3 & 21.9 \\
Angularity $a=+1$ & 10.3 & 20.0 & 15.8 & 24.5 & 12.0 & 19.3 & 14.0 & 21.6 \\
Det of Covariance Matrix & 11.2 & 21.2 & 18.1 & 27.0 & 9.4 & 20.9 & 13.5 & 24.6 \\
Track Spread: $\sqrt{<} p_{T}^{2}>/ p_{T}^{\text {jet }}$ & 16.5 & 25.3 & 16.5 & 25.3 & 9.3 & 20.1 & 9.3 & 20.1 \\
Track Count & 17.7 & 26.4 & 17.7 & 26.4 & 8.9 & 21.0 & 8.9 & 21.0 \\
Decluster with $k_{T}, \Delta R$ & 15.8 & 24.5 & 20.1 & 28.4 & 13.9 & 20.1 & 16.9 & 23.4 \\
Jet $m / p_{T}$ for $\mathrm{R}=0.3$ subjet & 13.1 & 25.9 & 16.3 & 27.7 & 11.9 & 24.2 & 14.8 & 26.2 \\
Planar Flow & 28.7 & 34.4 & 28.7 & 34.4 & 39.6 & 42.9 & 39.6 & 42.9 \\
Pull Magnitude & 37.0 & 39.0 & 32.9 & 35.6 & 30.6 & 30.2 & 29.6 & 30.6 \\
\hline Track Count \& Girth & 9.9 & 20.1 & 13.4 & 23.2 & 7.1 & 17.3 & $7.7^{*}$ & 18.7 \\
R=0.3 $m / p_{T} \& \mathrm{R}=0.7$ 2-Pt $\beta=1 / 5$ & $7.9^{*}$ & 17.7 & $12.2^{*}$ & 22.1 & 5.7 & $14.4^{*}$ & 8.5 & 17.9 \\
1-Subj $\beta=1 / 2 \& \mathrm{R}=0.72-\mathrm{Pt} \beta=1 / 5$ & 8.5 & $17.3^{*}$ & 12.9 & 22.1 & 6.0 & 14.6 & 8.6 & $17.7^{*}$ \\
Girth \& R=0.7 2-pt $\beta=1 / 10$ & 12.6 & 21.9 & 12.6 & $21.9^{*}$ & 9.2 & 18.0 & 9.2 & 18.0 \\
1-Subj $\beta=1 / 2 \&$ \&-Subj $\beta=1$ & 8.9 & 18.0 & 14.0 & 23.2 & $5.6^{*}$ & 15.0 & 8.4 & 18.4 \\
\hline Best Group of 3 & 7.5 & 17.0 & 11.0 & 20.9 & 4.7 & 14.0 & 6.9 & 16.6 \\
Best Group of 4 & 7.1 & 16.7 & 10.6 & 20.5 & 4.5 & 13.7 & 6.2 & 16.3 \\
Best Group of 5 & 6.9 & 16.4 & 10.4 & 20.0 & 4.3 & 13.3 & 6.1 & 15.9 \\
\hline
\end{tabular}

Table 1. Comparison of gluon efficiencies at the $50 \%$ quark acceptance working point. All of the single variables use $\mathrm{R}=0.5$ jets, whereas combinations sometimes include $\mathrm{R}=0.7$ jets. Gluon efficiencies, rather than gluon rejections (one minus efficiencies), are shown because a fractional improvement here is the same fractional improvement in $S / B$. Divided by two, it is also the fractional improvement in $S / \sqrt{B}$. These scores have $\pm 0.5 \%$ statistical errors, but they are correlated - the differences between variables has smaller spread, as does the improvement when combining variables. Because of the large number of variables and parameters, and the larger number of possible combinations of these, there is definitely a look-elsewhere-type effect when choosing the top pair. Many pairs statistically tied for the top spot in each category, so five pairs were chosen as representative. Their scores are marked with asterisks, as are the best individual variables in each category. The best groups of 3,4 , and 5 start to show diminishing returns. 


\section{References}

[1] C. T. Hill and S. J. Parke, Phys. Rev. D 49, 4454 (1994) [arXiv:hep-ph/9312324].

[2] B. Bellazzini, C. Csaki, A. Falkowski and A. Weiler, Phys. Rev. D 80, 075008 (2009) [arXiv:0906.3026 [hep-ph]].

[3] J. Gallicchio and M. D. Schwartz, JHEP 1110, 103 (2011) [arXiv:1104.1175 [hep-ph]].

[4] G. Dissertori, I.K. Knowles, M. Schmelling, Quantum Chromodynamics: High energy experiments and theory, International Series of Monographs on Physics No. 115, ISBN 0198505728, Oxford University Press, Feb. 2003. Reprinted in 2005.

[5] A. Capella, I. M. Dremin, J. W. Gary, V. A. Nechitailo and J. Tran Thanh Van, Phys. Rev. D 61, 074009 (2000) [arXiv:hep-ph/9910226].

[6] P. Bolzoni, B. A. Kniehl and A. V. Kotikov, arXiv:1209.5914 [hep-ph].

[7] K. Ackerstaff et al. [OPAL Collaboration], Eur. Phys. J. C 1, 479 (1998) [arXiv:hep-ex/9708029].

[8] R. Barate et al. [ALEPH Collaboration], Eur. Phys. J. C 17, 1 (2000).

[9] P. Abreu et al. [DELPHI Collaboration], Eur. Phys. J. C 4, 1 (1998).

[10] P. Abreu et al. [DELPHI Collaboration], Phys. Lett. B 401, 181 (1997).

[11] P. Abreu et al. [DELPHI Collaboration], Eur. Phys. J. C 17, 207 (2000) [arXiv:hep-ex/0106063].

[12] K. Ackerstaff et al. [OPAL Collaboration], Eur. Phys. J. C 8, 241 (1999) [arXiv:hep-ex/9805025].

[13] G. Abbiendi et al. [OPAL Collaboration], Eur. Phys. J. C 17, 373 (2000) [arXiv:hep-ex/0007017].

[14] D. Buskulic et al. [ALEPH Collaboration], Phys. Lett. B 384, 353 (1996).

[15] O. Biebel [OPAL Collaboration], ZPC 69, 543 (1996).

[16] B. Abbott et al. [D0 Collaboration], Phys. Rev. Lett. 83, 1908 (1999) [hep-ex/9901023].

[17] T. Aaltonen et al. [CDF Collaboration], Phys. Rev. D 81, 052011 (2010) [arXiv:1002.0365 [hep-ex]].

[18] S. Catani, F. Krauss, R. Kuhn and B. R. Webber, JHEP 0111, 063 (2001) [hep-ph/0109231].

[19] S. Hoeche, F. Krauss, N. Lavesson, L. Lonnblad, M. Mangano, A. Schalicke and S. Schumann, hep-ph/0602031.

[20] A. Banfi, G. P. Salam and G. Zanderighi, Eur. Phys. J. C 47, 113 (2006) [arXiv:hep-ph/0601139].

[21] J. Alwall et al., JHEP 0709, 028 (2007) [arXiv:0706.2334 [hep-ph]].

[22] T. Sjostrand, S. Mrenna and P. Z. Skands, Comput. Phys. Commun. 178, 852 (2008) [arXiv:0710.3820 [hep-ph]].

[23] M. Bahr et al., Eur. Phys. J. C 58, 639 (2008) [arXiv:0803.0883 [hep-ph]].

[24] M. Cacciari and G. P. Salam, Phys. Lett. B 641, 57 (2006) [arXiv:hep-ph/0512210]. 
[25] A. Hoecker et al, TMVA Toolkit for Multivariate Data Analysis with ROOT, http://tmva. sourceforge.net/.

[26] R. Brun and F. Rademakers, ROOT - An Object Oriented Data Analysis Framework, Proceedings AIHENP'96 Workshop, Lausanne, Sep. 1996, Nucl. Inst. \& Meth. in Phys. Res. A 389 (1997) 81-86. See also http://root.cern.ch/.

[27] E. Feng [ATLAS Collaboration], arXiv:1010.1974 [hep-ex].

[28] G. Aad et al. [ATLAS Collaboration], arXiv:1112.6426 [hep-ex].

[29] ATLAS-CONF-2011-073, May 2011.

[30] M. Dasgupta, K. Khelifa-Kerfa, S. Marzani and M. Spannowsky, JHEP 1210, 126 (2012) [arXiv:1207.1640 [hep-ph]].

[31] Y. T. Chien, R. Kelley, M. D. Schwartz and H. X. Zhu, arXiv:1208.0010 [hep-ph].

[32] D. Acosta et al. [CDF Collaboration], Phys. Rev. D 71, 112002 (2005) [arXiv:hep-ex/0505013].

[33] P. Kurt [CMS Collaboration], PoS 2008LHC, 102 (2008).

[34] P. Kurt [CMS Collaboration], "Jet shapes," CERN-CMS-CR-2009-005

[35] J. Ellis and N. E. Mavromatos, Phys. Lett. B 711, 139 (2012) [arXiv:1012.4353 [hep-th]].

[36] J. Gallicchio and M. D. Schwartz, Phys. Rev. Lett. 105, 022001 (2010) [arXiv:1001.5027 [hep-ph]].

[37] V. Gavrilov, O. Kodolova and N. Lychkovskaya [CMS Collaboration], J. Phys. G37, 075009 (2010).

[38] L. G. Almeida, S. J. Lee, G. Perez, G. F. Sterman, I. Sung and J. Virzi, Phys. Rev. D 79, 074017 (2009) [arXiv:0807.0234 [hep-ph]].

[39] C. F. Berger, T. Kucs and G. F. Sterman, Phys. Rev. D 68, 014012 (2003) [arXiv:hep-ph/0303051].

[40] J. Thaler and K. Van Tilburg, JHEP 1103, 015 (2011) [arXiv:1011.2268 [hep-ph]].

[41] I. Feige, M. D. Schwartz, I. W. Stewart and J. Thaler, Phys. Rev. Lett. 109, 092001 (2012) [arXiv:1204.3898 [hep-ph]].

[42] [D0 Collaboration], D0Note 6087-CONF, "Search for the standard model Higgs boson in the $Z H \rightarrow b b \nu \nu$ channel in $6.4 \mathrm{fb}-1$ of ppbar collisions at sqrt(s)=1.96 TeV", Preliminary Results for Summer 2010 Conferences, http://www-d0.fnal.gov/Run2Physics/WWW/results/prelim/HIGGS/H90/ August 2010.

[43] J. Gallicchio, J. Huth, M. Kagan, M. D. Schwartz, K. Black and B. Tweedie, JHEP 1104, 069 (2011) [arXiv:1010.3698 [hep-ph]].

[44] Y. Cui, Z. Han and M. D. Schwartz, Phys. Rev. D 83, 074023 (2011) [arXiv:1012.2077 [hep-ph]].

[45] [ATLAS Collaboration], ATLAS-CONF-2012-138. 